\title{
System of Spheres-based Multiple Contractions
}

\author{
Eduardo Fermé • Maurício D. L. Reis
}

Received: 2 October 2010 / Accepted: 1 May 2011 / Published online: 3 June 2011

(c) Springer Science+Business Media B.V. 2011

\begin{abstract}
We propose a new class of multiple contraction operations - the system of spheres-based multiple contractions — which are a generalization of Grove's system of spheres-based (singleton) contractions to the case of contractions by (possibly non-singleton) sets of sentences. Furthermore, we show that this new class of functions is a subclass of the class of the partial meet multiple contractions.
\end{abstract}

Keywords Belief change - Theory contraction • Multiple contraction • Possible worlds semantics $\cdot$ System of spheres

\section{Introduction}

The logic of theory change became a major subject in philosophical logic and artificial intelligence in the middle of the 1980's. The most important model of belief change is known as the AGM model and has been proposed by Alchourrón et al. [2]. The AGM model is a formal framework to characterize the dynamics and state of belief of a rational agent. In the AGM framework,

M. D. L. Reis was supported by Fundação para a Ciência e a Tecnologia (FCT) through the grant SFRH/BD/30175/2006 financed by national funds from Ministério da Ciência, Tecnologia e Ensino Superior (MCTES) from Portugal.

E. Fermé $(\bowtie) \cdot$ M. D. L. Reis

Madeira Interactive Technologies Institute, Universidade da Madeira, Funchal, Portugal e-mail: ferme@uma.pt

\section{D. L. Reis}

Centro de Ciências Exactas e da Engenharia, Universidade da Madeira, Funchal, Portugal e-mail: m_reis@uma.pt 
the beliefs are represented by belief sets (i.e. deductively closed sets of sentences). A change consists in adding or removing a specific sentence from a belief set to obtain a new belief set. The AGM model has been characterized in at least five different equivalent ways: postulates, partial meet functions, epistemic entrenchment, safe/kernel contraction and sphere-systems.

AGM has acquired the status of a standard model, which inspired many researchers to propose extensions and generalizations. One of those generalizations is multiple belief change which consists of the study of change operations for which the input is a (possibly non-singleton) set of sentences instead of a single sentence. In particular, in what concerns operations dealing with the removal of sets with more than one element from a theory we can find three different models in the literature: package contraction [4-6, 8] (the output of the contraction does not contain any of the elements of the input set), choice contraction [6] (at least one of the elements of the input set is not contained in the output of the contraction), and set contraction $[12,13]$ (the output of the contraction is consistent with the input set). In the present paper we will only consider package contraction and we will use the expression multiple contraction to refer to an operation of that kind. More precisely, we will only consider multiple contractions by finite sets. Hence, throughout this paper whenever we refer to the multiple contraction of a belief set $\mathbf{K}$ by a set of sentences $B$, such set $B$ is assumed to be finite. We will use the expression singleton contraction to designate contractions by a single sentence.

The first constructive model of multiple contractions to be presented has been the partial meet multiple contraction [6, 8]. Later in [3] kernel contraction has been generalized to the case of multiple contraction and in [10] a semantics for partial meet multiple contraction in terms of possible worlds has been presented.

In this work we aim to propose a way of defining system of spheres-based multiple contractions, assuring that such operations: (1) generalize Grove's system of spheres-based (singleton) contractions to the multiple contraction level, and (2) are partial meet multiple contractions.

\section{Background}

\subsection{Formal Preliminaries}

We will assume a language $\mathcal{L}$ that is closed under truth-functional operations. We shall make use of a consequence operation $C n$ that takes sets of sentences to sets of sentences and which satisfies the standard Tarskian properties, namely inclusion, monotony and iteration. Furthermore we will assume that Cn satisfies supraclassicality, compactness and deduction. We will sometimes use $C n(\alpha)$ for $C n(\{\alpha\}), A \vdash \alpha$ for $\alpha \in C n(A), \vdash \alpha$ for $\alpha \in C n(\emptyset), A \nvdash \alpha$ for $\alpha \notin C n(A), \not \forall \alpha$ for $\alpha \notin C n(\emptyset)$. The letters $\alpha, \alpha, \beta, \ldots$ (except for $\gamma$ ) will be used to denote sentences. $T$ stands for an arbitrary tautology and $\perp$ for an arbitrary contradiction. $A, A_{i}, B, \ldots$ shall denote subsets of sentences of $\mathcal{L}$. 
$\mathbf{K}$ is reserved to represent a set of sentences that is closed under logical consequence (i.e. $\mathbf{K}=C n(\mathbf{K}))$ - such a set is called a belief set or theory. The conjunction of all elements of a finite and non-empty set $A$ shall be denoted by $\& A$. We shall denote the set of all maximal consistent subsets of $\mathcal{L}$ by $\mathcal{M}_{\mathcal{L}}$. We will use the expression possible world (or just world) to designate an element of $\mathcal{M}_{\mathcal{L}} \cdot \mathcal{M}, \mathcal{N}_{i}, \mathcal{W}, \ldots$, (except for $\mathcal{L}$ and $\mathcal{P}$ ), shall be used to denote subsets of $\mathcal{M}_{\mathcal{L}}$. Such sets are called propositions. Given a set of sentences $R$, the set consisting of all the possible worlds that contain $R$ is denoted by $\|R\|$. The elements of $\|R\|$ are the $R$-worlds. $\|\varphi\|$ is an abbreviation of $\|\{\varphi\}\|$ and the elements of $\|\varphi\|$ are the $\varphi$-worlds. To any set of possible worlds $\mathcal{V}$ we associate a belief set $T h(\mathcal{V})$ given by $T h(\mathcal{V})=\bigcap \mathcal{V}$ - under the assumption that $\bigcap \emptyset=\mathcal{L}$. $\mathbb{M}, \mathbb{N}_{i}, \mathbb{W}, \ldots$ shall be used to denote subsets of $\mathcal{P}\left(\mathcal{M}_{\mathcal{L}}\right)$.

\subsection{Partial Meet Contractions}

We start by recalling the basic concepts necessary for the definition of the partial meet contractions introduced in [2].

Given a belief set $\mathbf{K}$ and a set of sentences $B$, the remainder set of $\mathbf{K}$ by $B$ is the set of maximal subsets of $\mathbf{K}$ that do not imply any element of $B$ and is denoted by $\mathbf{K} \perp B$. The elements of $\mathbf{K} \perp B$ are the remainders (of $\mathbf{K}$, by $B$ ) [1, p. 128]. It is also convenient to notice here that, according to [1, Observation 2.2], since we are assuming that the consequence operation $C n$ is compact, it holds that $\mathbf{K} \perp B \neq \emptyset$ if and only if $B \cap C n(\emptyset)=\emptyset$.

For any sentence $\alpha, \mathbf{K} \perp \alpha$ is an abbreviation of $\mathbf{K} \perp\{\alpha\}$ and is called the remainder set of $\mathbf{K}$ by $\alpha$.

Definition 2.1 [2] Let $\mathbf{K}$ be a belief set. A selection function for $\mathbf{K}$ is a function $\gamma$ such that for all sentences $\alpha$ : if $\mathbf{K} \perp \alpha \neq \emptyset$ then $\emptyset \neq \gamma(\mathbf{K} \perp \alpha) \subseteq \mathbf{K} \perp \alpha$, and if $\mathbf{K} \perp \alpha=\emptyset$, then $\gamma(\mathbf{K} \perp \alpha)=\{\mathbf{K}\}$.

An operation - is a partial meet contraction on $\mathbf{K}$ if and only if there is a selection function $\gamma$ for $\mathbf{K}$ such that for all sentences $\alpha: \mathbf{K}-\alpha=\bigcap \gamma(\mathbf{K} \perp \alpha)$.

\subsection{System of Spheres-based Contractions}

Now we recall the definitions of a system of spheres and of the system of spheres-based contractions presented in [7].

Definition 2.2 [7] Let $\mathcal{X}$ be a subset of $\mathcal{M}_{\mathcal{L}}$. A system of spheres (abrev. S.S.), or spheres' system, centred on $\mathcal{X}$ is a collection $\mathbb{S}$ of subsets of $\mathcal{M}_{\mathcal{L}}$, i.e. $\mathbb{S} \subseteq$ $\mathcal{P}\left(\mathcal{M}_{\mathcal{L}}\right)$, that satisfies the following conditions:

(S1) $\mathbb{S}$ is totally ordered with respect to set inclusion; that is, if $\mathcal{U}, \mathcal{V} \in \mathbb{S}$, then $\mathcal{U} \subseteq \mathcal{V}$ or $\mathcal{V} \subseteq \mathcal{U}$

(S2) $\mathcal{X} \in \mathbb{S}$, and if $\mathcal{U} \in \mathbb{S}$ then $\mathcal{X} \subseteq \mathcal{U}$. 
(S3) $\mathcal{M}_{\mathcal{L}} \in \mathbb{S}$ (and so it is the largest element of $\mathbb{S}$ ).

$(\mathbb{S} 4)$ For every $\varphi \in \mathcal{L}$, if there is any element in $\mathbb{S}$ intersecting $\|\varphi\|$ then there is also a smallest element in $\mathbb{S}$ intersecting $\|\varphi\|$.

The elements of $\mathbb{S}$ are called spheres.

For any consistent sentence $\varphi \in \mathcal{L}$, the smallest sphere in $\mathbb{S}$ intersecting $\|\varphi\|$ is denoted by $\mathbb{S}_{\varphi}$ and $f_{\mathbb{S}}(\varphi)$ denotes the set consisting of the $\varphi$-worlds closest to $\mathcal{X}$, i.e.,

$$
f_{\mathbb{S}}(\varphi)=\|\varphi\| \cap \mathbb{S}_{\varphi}
$$

Definition 2.3 [7] Let $\mathbf{K}$ be a belief set and $\mathbb{S}$ be a system of spheres centred on $\|\mathbf{K}\|$. The $\mathbb{S}$-based contraction on $\mathbf{K}$ is the contraction operation $-_{\mathbb{S}}$ defined, for any $\varphi \in \mathcal{L}$, by:

$$
\mathbf{K}-\mathbb{S} \varphi= \begin{cases}T h\left(\|\mathbf{K}\| \cup f_{\mathbb{S}}(\neg \varphi)\right), & \text { if } \nvdash \varphi \\ \mathbf{K} & \text { if } \vdash \varphi .\end{cases}
$$

An operation - on $\mathbf{K}$ is a system of spheres-based contraction on $\mathbf{K}$ if and only if there is a system of spheres $\mathbb{S}$ centred on $\|\mathbf{K}\|$, such that, for all sentences $\varphi \in \mathcal{L}, \mathbf{K}-\varphi=\mathbf{K}-{ }_{\mathbb{S}} \varphi$.

To close this subsection we remind that Grove [7] has shown that every system of spheres-based contraction is a partial meet contraction. More precisely, in [7] it has been shown that the class of system of spheres-based contractions coincides with the class of transitively relational partial meet contractions (TRPMCs), introduced in [2], which is a (proper) subclass of the class of partial meet contractions.

\subsection{Partial Meet Multiple Contractions}

The partial meet multiple contractions are a generalization of the partial meet contraction functions to the case of contractions by (possibly non-singleton) sets.

Definition $2.4[6,8]$ Let $\mathbf{K}$ be a belief set. A package selection function for $\mathbf{K}$ is a function $\gamma$ such that for all sets of sentences $B$ : if $\mathbf{K} \perp B \neq \emptyset$, then $\emptyset \neq$ $\gamma(\mathbf{K} \perp B) \subseteq \mathbf{K} \perp B$, and if $\mathbf{K} \perp B=\emptyset$ then $\gamma(\mathbf{K} \perp B)=\{\mathbf{K}\}$.

An operation $\div$ is a partial meet multiple contraction on $\mathbf{K}$ if and only if there is some package selection function $\gamma$ for $\mathbf{K}$, such that for all sets of sentences $B: \mathbf{K} \div B=\bigcap \gamma(\mathbf{K} \perp B)$.

An operation $\div$ on $\mathbf{K}$ is a maxichoice multiple contraction if and only if it is a partial meet multiple contraction generated by a package selection function $\gamma$ such that for all sets $B$, the set $\gamma(\mathbf{K} \perp B)$ has exactly one element. The full meet multiple contraction on $\mathbf{K}$ is the partial meet multiple contraction $\dot{\sim}$ that is generated by the package selection function $\gamma$ such that for all sets $B$, 
if $\mathbf{K} \perp B \neq \emptyset$, then $\gamma(\mathbf{K} \perp B)=\mathbf{K} \perp B$, i.e., $\mathbf{K} \dot{\sim} B=\bigcap \mathbf{K} \perp B$, if $B \cap C n(\emptyset)=\emptyset$, and $\mathbf{K} \dot{\sim} B=\mathbf{K}$, otherwise.

\subsection{Possible Worlds Semantics for Partial Meet Multiple Contractions}

In [10] we have presented the possible worlds semantics for partial meet multiple contraction. More precisely, in that paper we have shown how the remainders can be defined in terms of possible worlds and, making use of that alternative way of defining the remainders, we have shown how Partial Meet Multiple Contractions can be defined as intersections of (appropriate) sets of possible worlds.

In the present subsection we will recall the concepts and results of the mentioned paper which we will need further ahead.

We start by recalling the definition of the set $\mathbb{W}_{\mathbf{K} \perp B}$, that allows us to define a remainder set in terms of possible worlds.

Definition 2.5 [10] Let $\mathbf{K}$ be a belief set and $B$ be a set of sentences. We denote by $\mathbb{W}_{\mathbf{K} \perp B}$ the subset of $\mathcal{P}\left(\bigcup\left\{\left\|\neg \alpha_{i}\right\|: \alpha_{i} \in B \cap \mathbf{K}\right\}\right)$ such that $\mathcal{W} \in \mathbb{W}_{\mathbf{K} \perp B}$ if and only if:

1. $\mathcal{W} \cap\left\|\neg \alpha_{i}\right\| \neq \emptyset$, for all $\alpha_{i} \in B \cap \mathbf{K}$.

2. If $M \in \mathcal{W}$ then there is some $\alpha_{j} \in B \cap \mathbf{K}$ such that $\mathcal{W} \cap\left\|\neg \alpha_{j}\right\|=\{M\}$.

The relation between the set $\mathbb{W}_{\mathbf{K} \perp B}$ and the remainder set $\mathbf{K} \perp B$ is the following:

Observation 2.6 [10] Let $\mathbf{K}$ be a belief set and $B$ be a finite set of sentences. Then:

1. If $\mathcal{W} \in \mathbb{W}_{\mathbf{K} \perp B}$ then $X=T h(\|\mathbf{K}\| \cup \mathcal{W}) \in \mathbf{K} \perp B$.

2. If $X \in \mathbf{K} \perp B$ then there is some $\mathcal{W} \in \mathbb{W}_{\mathbf{K} \perp B}$ such that $X=T h(\|\mathbf{K}\| \cup \mathcal{W})$.

3. $\mathbf{K} \perp B=\left\{T h(\|\mathbf{K}\| \cup \mathcal{W}): \mathcal{W} \in \mathbb{W}_{\mathbf{K} \perp B}\right\}$.

The first statement of the observation means that the sets in $\mathbb{W}_{\mathbf{K} \perp B}$ are minimal sets of worlds in the sense that the addition of each of those sets to $\|\mathbf{K}\|$ corresponds to a maximal subset of $\mathbf{K}$ that does not imply any element of $B$. The second statement yields that each of the remainders can be constructed by means of a set of $\mathbb{W}_{\mathbf{K} \perp B}$. The third shows how to construct a remainder set in terms of possible worlds.

Using the set $\mathbb{W}_{\mathbf{K} \perp B}$ it is possible to define a partial meet multiple contraction in terms of possible worlds:

Observation 2.7 [10] Let $\mathbf{K}$ be a belief set. An operation $\div$ is a partial meet multiple contraction on $\mathbf{K}$ if and only if for any set of sentences $B$ :

$$
\mathbf{K} \div B=\operatorname{Th}\left(\|\mathbf{K}\| \cup\left(\bigcup f\left(\mathbb{W}_{\mathbf{K} \perp B}\right)\right)\right),
$$


where $f$ is a propositional package selection function for $\|\mathbf{K}\|$ such that for all sets of sentences $B$ : (1) $f\left(\mathbb{W}_{\mathbf{K} \perp B}\right) \subseteq \mathbb{W}_{\mathbf{K} \perp B}$, and (2) if $\mathbb{W}_{\mathbf{K} \perp B} \neq \emptyset$ then $f\left(\mathbb{W}_{\mathbf{K} \perp B}\right) \neq \emptyset$.

The lower bound of partial meet multiple contraction, called full meet multiple contraction corresponds to the situation when $f\left(\mathbb{W}_{\mathbf{K} \perp B}\right)=\mathbb{W}_{\mathbf{K} \perp B}[10$, Observation 3.6]. In the case of singleton contraction, the full meet contraction $\mathbf{K} \sim \alpha$ corresponds to the addition of the whole set $\|\neg \alpha\|$ to $\|\mathbf{K}\|$. However, in the multiple case it may not hold that $\bigcup \mathbb{W}_{\mathbf{K} \perp B}=\bigcup\left\{\left\|\neg \alpha_{i}\right\|: \alpha_{i} \in B \cap \mathbf{K}\right\}$ [10, Observation 3.7]. For instance, if $B=\{\alpha, \alpha \wedge \beta\}$ and $\alpha \nvdash \beta$, then since every $\neg \alpha$-world is also a $\neg(\alpha \wedge \beta)$-world the addition of a $\neg \alpha$-world to $\|\mathbf{K}\|$ immediately leads to the removal of both $\alpha$ and $\alpha \wedge \beta$ from the new (resulting) belief set. Therefore none of the $\alpha \wedge \neg \beta$-worlds (i.e. the worlds in $\|\neg(\alpha \wedge \beta)\| \backslash$ $\|\neg \alpha\|)$ belongs to a set $\mathcal{W}$ of $\mathbb{W}_{\mathbf{K} \perp B}$. Based on this fact in [10, Observation 3.9] the following equality has been presented

$$
\bigcup \mathbb{W}_{\mathbf{K} \perp B}=\bigcup\left\{\left\|\neg \alpha_{i}\right\|: \alpha_{i} \in B_{N} \cap \mathbf{K}\right\},
$$

where

$$
B_{N}=\left\{\alpha_{i} \in B: \text { For all } \alpha_{j} \in B \text { it holds that }\left\|\neg \alpha_{j}\right\| \not \subset\left\|\neg \alpha_{i}\right\|\right\} .^{1}
$$

Furthermore, making use of the above equality the following observation has been obtained in the mentioned paper:

Observation 2.8 [10] Let $\mathbf{K}$ be a belief set and $B$ be a set of sentences and $\dot{\sim}$ be the full meet multiple contraction on $\mathbf{K}$. Then

$$
\|\mathbf{K} \dot{\sim} B\|=\|\mathbf{K}\| \cup\left(\bigcup\left\{\left\|\neg \alpha_{i}\right\|: \alpha_{i} \in B_{N} \cap \mathbf{K}\right\}\right) .
$$

\section{How (and how not) to Construct Partial Meet Multiple Contractions Using Systems of Spheres}

In this section we will generalize Grove's construction of a S.S.-based contraction to the multiple case. More precisely, given a belief set $\mathbf{K}$ and a system of spheres $\mathbb{S}$ centred on $\|\mathbf{K}\|$ we will define a multiple contraction operation $\div \mathbb{S}$ on $\mathbf{K}$ - which we will designate by $\mathbb{S}$-based multiple contraction on $\mathbf{K}-$ such that (1) it is a partial meet multiple contraction, and (2) it is such that for all sentences $\alpha \in \mathcal{L}$ it holds that $\mathbf{K} \div \mathbb{s}\{\alpha\}=\mathbf{K}-\mathbb{s} \alpha$, where ${ }_{\mathbb{S}}$ is the $\mathbb{S}$-based (singleton) contraction.

We will start by showing that the most intuitive proposals for the definition of the $\mathbb{S}$-based multiple contraction fail to be partial meet multiple contractions,

\footnotetext{
${ }^{1}$ Notice that the set $B_{N}$ can, alternatively, be defined without making use of the notion of possible world, in the following (equivalent) way:

$$
B_{N}=\left\{\alpha_{i} \in B: \alpha_{i} \not \nvdash \alpha_{j} \text { for all } \alpha_{j} \in B \text { s.t. } \operatorname{Cn}\left(\alpha_{i}\right) \neq \operatorname{Cn}\left(\alpha_{j}\right)\right\} .
$$
}


then we find sufficient conditions for a $\mathbb{S}$-based multiple contraction on $\mathbf{K}$ be a partial meet multiple contraction and finally we present a method to construct functions that satisfy those conditions.

Recalling that if $\div$ is a partial meet multiple contraction and $B$ contains tautologies (i.e. $B \cap C n(\emptyset) \neq \varnothing$ ) then $\mathbf{K} \div B=\mathbf{K}$, we immediately conclude that the operation $\div_{\mathbb{S}}$ must satisfy $\mathbf{K} \div{ }_{\mathbb{S}} B=\mathbf{K}$, for all such sets $B$.

Having seen this, it only remains to investigate how shall $\mathbf{K} \div \mathbb{S} B$ be defined for sets $B$ such that $B \cap C n(\emptyset)=\emptyset$. However, as we will see below, it is not trivial to find out an appropriate way of defining $\mathbf{K} \div \mathbb{S} B$ (in order to assure that $\div$ s fulfils the desired properties (1) and (2) introduced above). Indeed, in what follows we show that the most intuitive proposals for the definition of the S-based multiple contraction on $\mathbf{K}$ actually do not fulfil the above mentioned requirements.

\subsection{Unsuccessful Proposals}

\subsubsection{First Unsuccessful Proposal}

Consider a belief set $\mathbf{K}$ and a system of spheres $\mathbb{S}$ centred on $\|\mathbf{K}\|$. Recalling that Grove's $\mathbb{S}$-based singleton contraction is such that if $\forall \varphi$ then $\mathbf{K}-{ }_{\mathbb{S}} \varphi=$ $T h\left(\|\mathbf{K}\| \cup f_{\mathbb{S}}(\neg \varphi)\right)$, it follows that the operation $\div_{\mathbb{S}}^{1}$ defined by:

$$
\mathbf{K} \div{ }_{\mathbb{S}}^{1} B= \begin{cases}T h\left(\|\mathbf{K}\| \cup\left(\cup_{\alpha_{i} \in B} f_{\mathbb{S}}\left(\neg \alpha_{i}\right)\right)\right) & \text { if } B \cap C n(\emptyset)=\emptyset \\ \mathbf{K} & \text { if } B \cap C n(\emptyset) \neq \varnothing\end{cases}
$$

is the most obvious proposal for the definition of the $\mathbb{S}$-based multiple contraction on K. ${ }^{2,3}$ However, the following simple counterexample, shows that $\div_{\mathbb{S}}^{1}$ is not a partial meet multiple contraction.

Counterexample 3.1 Consider Fig. 1 and let $B=\left\{\alpha_{1}, \alpha_{2}, \alpha_{3}\right\}$. Notice that, in these conditions, it follows from Definition 2.2 that $f_{\mathbb{S}}\left(\neg \alpha_{1}\right)=\left\|\neg \alpha_{1}\right\|$, $f_{\mathbb{S}}\left(\neg \alpha_{2}\right)=\left\|\neg \alpha_{2}\right\|$ and $f_{\mathbb{S}}\left(\neg \alpha_{3}\right)=\left\|\neg \alpha_{3}\right\|$. Then, according to the definition of $\div{ }_{\mathbb{S}}^{1}$ proposed above, we have that $\mathbf{K} \div{ }_{\mathbb{S}}^{1} B=\bigcap\left(\|\mathbf{K}\| \cup\left\|\neg \alpha_{1}\right\| \cup\left\|\neg \alpha_{2}\right\| \cup\left\|\neg \alpha_{3}\right\|\right)$, that is, $\mathbf{K} \div{ }_{\mathbb{S}}^{1} B$ consists of the intersection of all the worlds included in the shaded region of Fig. 1. Then, in particular, $\left\|\neg \alpha_{1}\right\| \subseteq\left\|\mathbf{K} \div_{\mathbb{S}}^{1} B\right\|$. On the other hand, in

\footnotetext{
${ }^{2}$ Notice that, since, whenever $B \cap C n(\emptyset)=\emptyset$ it holds that $\bigcap_{\alpha_{i} \in B} \mathbf{K}-\mathbb{S} \alpha_{i}=\bigcap_{\alpha_{i} \in B}(T h(\|\mathbf{K}\| \cup$ $\left.\left.f_{\mathbb{S}}\left(\neg \alpha_{i}\right)\right)\right)=\bigcap_{\alpha_{i} \in B}\left(\bigcap\left(\|\mathbf{K}\| \cup f_{\mathbb{S}}\left(\neg \alpha_{i}\right)\right)\right)=\bigcap\left(\|\mathbf{K}\| \cup\left(\cup_{\alpha_{i} \in B} f_{\mathbb{S}}\left(\neg \alpha_{i}\right)\right)\right)=\operatorname{Th}\left(\|\mathbf{K}\| \cup\left(\cup_{\alpha_{i} \in B}\right.\right.$ $\left.f_{\mathbb{S}}\left(\neg \alpha_{i}\right)\right)$ ), it follows that operation $\div_{\mathbb{S}}^{1}$ on $\mathbf{K}$ can be equivalently defined in the following (alternative) way:

$$
\mathbf{K} \div{ }_{\mathbb{S}}^{1} B= \begin{cases}\bigcap_{\alpha_{i} \in B} \mathbf{K}-\mathbb{S} \alpha_{i} & \text { if } B \cap C n(\emptyset)=\emptyset \\ \mathbf{K} & \text { if } B \cap C n(\emptyset) \neq \emptyset\end{cases}
$$

where $-\mathbb{S}$ is the $\mathbb{S}$-based (singleton) contraction.

${ }^{3}$ Based on a ranking theoretic approach Spohn [11] suggested a multiple contraction function very similar to operation $\div \stackrel{1}{\mathbb{S}}$.
} 
Fig. 1 Illustration of Counterexample 3.1

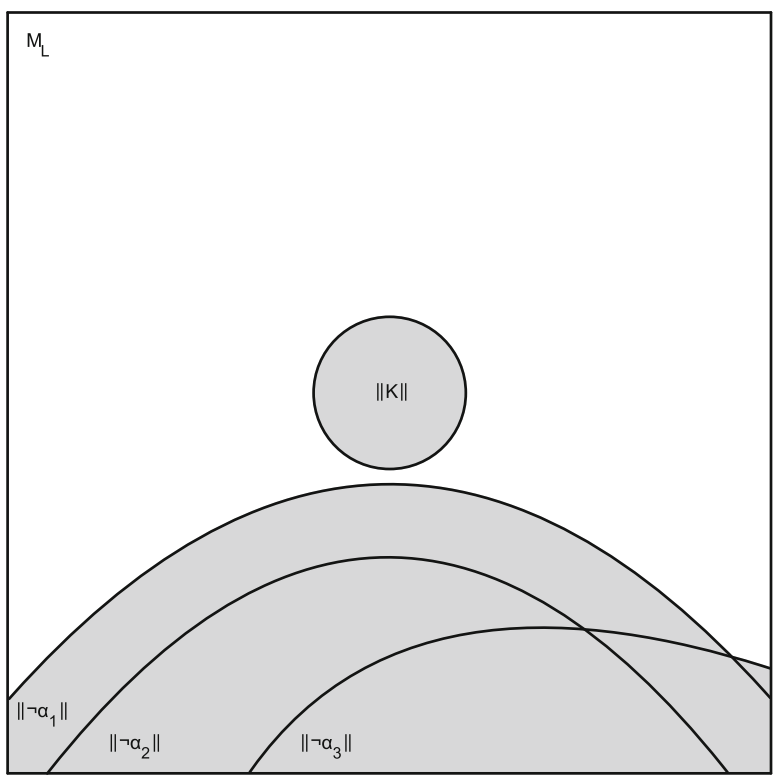

the above conditions, $B_{N}=\left\{\alpha_{2}, \alpha_{3}\right\}$, from which it follows (by Observation 2.8) that $\mathbf{K} \div{ }_{\mathbb{S}}^{1} B$ is not a superset of the full meet multiple contraction, and therefore $\div{ }_{\mathbb{S}}^{1}$ is not a partial meet multiple contraction.

\subsubsection{Second Unsuccessful Proposal}

Taking into account that $\div{ }_{\mathbb{S}}^{1}$ failled to be a partial meet multiple contraction because that $\mathbf{K} \div{ }_{\mathbb{S}}^{1} B$ was not a superset of the full meet multiple contraction, in our second proposal we just replace $B$ by $B_{N}$ :

$$
\mathbf{K} \div{ }_{\mathbb{S}}^{2} B= \begin{cases}T h\left(\|\mathbf{K}\| \cup\left(\bigcup_{\alpha_{i} \in B_{N}} f_{\mathbb{S}}\left(\neg \alpha_{i}\right)\right)\right) & \text { if } B \cap C n(\emptyset)=\varnothing \\ \mathbf{K} & \text { if } B \cap C n(\emptyset) \neq \emptyset\end{cases}
$$

Nevertheless, the following counterexample shows that $\div_{\mathbb{S}}^{2}$ is not a partial meet multiple contraction either.

Counterexample 3.2 Consider Fig. 2 and let $B=\left\{\alpha_{1}, \alpha_{2}, \alpha_{3}\right\}$. Assume, additionally, that $\left\|T h\left(\|\mathbf{K}\| \cup f_{\mathbb{S}}\left(\neg \alpha_{2}\right) \cup f_{\mathbb{S}}\left(\neg \alpha_{3}\right)\right)\right\|=\|\mathbf{K}\| \cup f_{\mathbb{S}}\left(\neg \alpha_{2}\right) \cup f_{\mathbb{S}}\left(\neg \alpha_{3}\right)$ (which is the set represented by the shaded region of Fig. 2).

Notice that, in the above conditions, $B_{N}=\left\{\alpha_{2}, \alpha_{3}\right\}$. Therefore $\left\|\mathbf{K} \div{ }_{\mathbb{S}}^{2} B\right\|=$ $\|\mathbf{K}\| \cup f_{\mathbb{S}}\left(\neg \alpha_{2}\right) \cup f_{\mathbb{S}}\left(\neg \alpha_{3}\right)$.

Now assume by reductio that the operation $\div_{\mathbb{S}}^{2}$ is a partial meet multiple contraction on $\mathbf{K}$. Then, according to Observation 2.7, there is some $\mathbb{M}_{B} \subseteq \mathbb{W}_{\mathbf{K} \perp B}$ such that $\mathbb{M}_{B} \neq \emptyset$ and $\mathbf{K} \div 2=\operatorname{Ts}\left(\|\mathbf{K}\| \cup\left(\cup \mathbb{M}_{B}\right)\right)$. Consequently, $\|\mathbf{K}\| \cup$ $\left(\cup \mathbb{M}_{B}\right) \subseteq\left\|\mathbf{K} \div{ }_{\mathbb{S}}^{2} B\right\|$ which is to say that $\|\mathbf{K}\| \cup\left(\cup \mathbb{M}_{B}\right) \subseteq\|\mathbf{K}\| \cup f_{\mathbb{S}}\left(\neg \alpha_{2}\right) \cup$ $f_{\mathbb{S}}\left(\neg \alpha_{3}\right)$. Moreover, noticing that from $\mathbb{M}_{B} \subseteq \mathbb{W}_{\mathbf{K} \perp B}$ it follows that $\left(\bigcup \mathbb{M}_{B}\right) \cap$ 
Fig. 2 Illustration of Counterexample 3.2

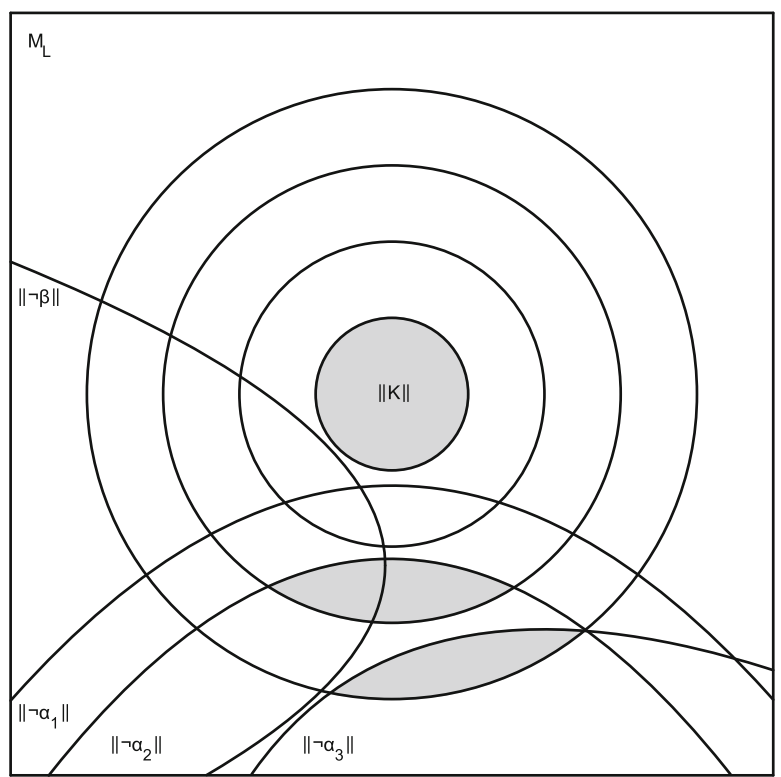

$\|K\|=\varnothing$ (since, according to Definition 2.5, $\left.\left(\bigcup \mathbb{W}_{\mathbf{K} \perp B}\right) \cap\|K\|=\emptyset\right)$, we can additionally conclude that $\left(\cup \mathbb{M}_{B}\right) \subseteq f_{\mathbb{S}}\left(\neg \alpha_{2}\right) \cup f_{\mathbb{S}}\left(\neg \alpha_{3}\right)$.

Furthermore, an attentive observation of Fig. 2 allows us to conclude that if $M \in f_{\mathbb{S}}\left(\neg \alpha_{2}\right)$ then it follows immediately from Definition 2.5 that there is no $\mathcal{W} \in \mathbb{W}_{\mathbf{K} \perp B}$ such that $\mathcal{W} \subseteq f_{\mathbb{S}}\left(\neg \alpha_{2}\right) \cup f_{\mathbb{S}}\left(\neg \alpha_{3}\right)$ and $M \in \mathcal{W}$. Therefore $\left(\bigcup \mathbb{M}_{B}\right) \subseteq f_{\mathbb{S}}\left(\neg \alpha_{3}\right)$.

Then, observing Fig. 2, from $\mathbf{K} \div{ }_{\mathbb{S}}^{2} B=T h\left(\|\mathbf{K}\| \cup\left(\cup \mathbb{M}_{B}\right)\right)$ we can conclude that $\beta \in \mathbf{K} \div{ }_{\mathbb{S}}^{2} B$. However, on the other hand, since $\mathbf{K} \div{ }_{\mathbb{S}}^{2} B=\bigcap(\|\mathbf{K}\| \cup$ $\left.f_{\mathbb{S}}\left(\neg \alpha_{2}\right) \cup f_{\mathbb{S}}\left(\neg \alpha_{3}\right)\right)$, also by analysing the mentioned figure, we can see that $\beta \notin \mathbf{K} \div{ }_{\mathbb{S}}^{2}$ B. From this contradiction we can conclude that $\div_{\mathbb{S}}^{2}$ is not a partial meet multiple contraction.

\subsubsection{Third Unsuccessful Proposal}

Having in mind Counterexample 3.2, we may notice that, if $B=\left\{\alpha_{i}, \alpha_{j}\right\} \subset \mathbf{K}$ and $\alpha_{i}, \alpha_{j}$ are such that $f_{\mathbb{S}}\left(\neg \alpha_{i}\right) \subset\left\|\neg \alpha_{j}\right\|$ and $f_{\mathbb{S}}\left(\neg \alpha_{j}\right) \backslash f_{\mathbb{S}}\left(\neg \alpha_{i}\right) \neq \emptyset$ then, in order to assure that an operation $\div_{\mathbb{S}}$ is a partial meet multiple contraction, we must prevent that the worlds in $f_{\mathbb{S}}\left(\neg \alpha_{j}\right) \backslash f_{\mathbb{S}}\left(\neg \alpha_{i}\right)$ are included in the set of possible worlds that we choose to intersect to originate $\mathbf{K} \div{ }_{\mathbb{S}} B$.

The above paragraph naturally leads us to consider the following alternative operation $\div 3$ :

$$
\mathbf{K} \div{ }_{\mathbb{S}}^{3} B= \begin{cases}\operatorname{Th}\left(\|\mathbf{K}\| \cup\left(\bigcup_{\alpha_{i} \in B_{\mathbb{S}, 1}} f_{\mathbb{S}}\left(\neg \alpha_{i}\right)\right)\right) & \text { if } B \cap \operatorname{Cn}(\emptyset)=\varnothing \\ \mathbf{K} & \text { if } B \cap \operatorname{Cn}(\emptyset) \neq \varnothing\end{cases}
$$


Fig. 3 Illustration of Counterexample 3.3

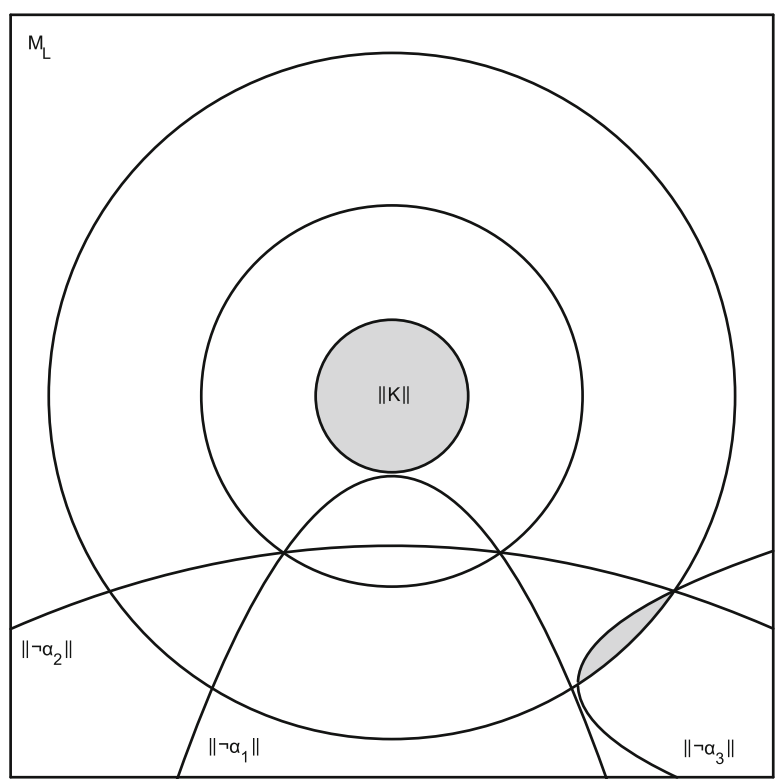

where, $\quad B_{\mathbb{S}, 1}=\left\{\alpha_{i} \in B: \forall \alpha_{k} \in B \backslash \operatorname{Cn}(\emptyset)\left(f_{\mathbb{S}}\left(\neg \alpha_{k}\right)=f_{\mathbb{S}}\left(\neg \alpha_{i}\right)\right.\right.$ or $f_{\mathbb{S}}\left(\neg \alpha_{k}\right) \not \subset$ $\left.\left.\left\|\neg \alpha_{i}\right\|\right)\right\}^{4}$

Nonetheless, this construction also fails to be a partial meet multiple contraction, as we can conclude from the following counterexample:

Counterexample 3.3 Consider Fig. 3 and let $B=\left\{\alpha_{1}, \alpha_{2}, \alpha_{3}\right\}$. Notice that, in the above conditions we obtain that $B_{\mathbb{S}, 1}=\left\{\alpha_{3}\right\}$, and consequently, $\mathbf{K} \div{ }_{\mathbb{S}}^{3} B=$ $T h\left(\|\mathbf{K}\| \cup f_{\mathbb{S}}\left(\neg \alpha_{3}\right)\right)$. Hence, we have that $\mathbf{K} \div{ }_{\mathbb{S}}^{3} B$ consists of the intersection of all the worlds included in the shaded region of Fig. 3. So, noticing that none of those worlds is a $\neg \alpha_{1}$-world we immediately conclude that $\alpha_{1} \in \mathbf{K} \div{ }_{\mathbb{S}}^{3} B$ which contradicts the successful elimination of all the members of $B$ from $\mathbf{K}$, a primary condition of partial meet multiple contraction.

\section{2 (Finding) The Way to a Successful Proposal}

We will use the previous counterexamples in order to, finally, present a way of defining an operation $\div \mathbb{s}$ which fulfils the desired properties.

We start by observing that all the above proposed operations $\div_{\mathbb{S}}^{1}, \div_{\mathbb{S}}^{2}$ and $\div_{\mathbb{S}}^{3}$ have the form $T h\left(\|\mathbf{K}\| \cup\left(\bigcup_{\alpha_{i} \in B^{\prime}} f_{\mathbb{S}}\left(\neg \alpha_{i}\right)\right)\right)$ where $B^{\prime}$ is a subset of $B$. However we have shown that none of those operations is a partial meet multiple contraction.

\footnotetext{
${ }^{4}$ Notice that, if $B \subseteq(\mathbf{K} \backslash C n(\emptyset))$ and $\mathbb{S}=\left\{\|\mathbf{K}\|, \mathcal{M}_{\mathcal{L}}\right\}$ then $B_{\mathbb{S}, 1}=B_{N}$ (since, in that case, $\forall \alpha_{k} \in$ $\left.\mathbf{K} \backslash \operatorname{Cn}(\emptyset) f_{\mathbb{S}}\left(\neg \alpha_{k}\right)=\left\|\neg \alpha_{k}\right\|\right)$. However, in general, neither $B_{\mathbb{S}, 1} \subseteq B_{N}$ nor $B_{N} \subseteq B_{\mathbb{S}, 1}$. 
In particular, in $\div_{\mathbb{S}}^{2}$ we have taken $B^{\prime}=B_{N}$ and in $\dot{\leftarrow}_{\mathbb{S}}^{3}$ we have considered $B^{\prime}=B_{\mathbb{S}, 1}$, where the set $B_{\mathbb{S}, 1}$ has been defined with the specific goal of assuring that the set $\|\mathbf{K}\| \cup\left(\bigcup_{\alpha_{i} \in B_{\mathbb{S}, 1}} f_{\mathbb{S}}\left(\neg \alpha_{i}\right)\right)$ is a subset of $\|\mathbf{K}\| \cup\left(\bigcup_{\alpha_{i} \in B_{N}} f_{\mathbb{S}}\left(\neg \alpha_{i}\right)\right)$. However, it follows from Counterexample 3.3 that operation $\dot{\div}_{\mathbb{S}}^{3}$ fails to be a partial meet multiple contraction because the set $\|\mathbf{K}\| \cup\left(\bigcup_{\alpha_{i} \in B_{\mathbb{S}, 1}} f_{\mathbb{S}}\left(\neg \alpha_{i}\right)\right)$ has, in general, too few worlds.

Hence, holding on to the idea of defining $\mathbf{K} \div \mathbb{S} B$ as an intersection of a set of possible worlds of the form $\|\mathbf{K}\| \cup\left(\bigcup_{\alpha_{i} \in B^{\prime}} f_{\mathbb{S}}\left(\neg \alpha_{i}\right)\right)$ with $B^{\prime} \subseteq B$, our next goal is to find a systematic way of defining, a subset $B_{\mathbb{S}} \subseteq B$ such that: (i) $B_{\mathbb{S}, 1} \subseteq B_{\mathbb{S}} \subseteq B_{N}$ and (ii) the operation $\div{ }_{\mathbb{S}}$ defined by $\mathbf{K} \div{ }_{\mathbb{S}} B=$ $T h\left(\|\mathbf{K}\| \cup\left(\bigcup_{\alpha_{i} \in B_{\mathbb{S}}} f_{\mathbb{S}}\left(\neg \alpha_{i}\right)\right)\right)$ is a partial meet multiple contraction on $\mathbf{K}$.

So, in order to find out how to define a set $B_{\mathbb{S}} \subseteq B$ such that the above conditions hold, it is convenient to start by understanding in more detail why the set $\|\mathbf{K}\| \cup\left(\bigcup_{\alpha_{i} \in B_{\mathbb{S}, 1}} f_{\mathbb{S}}\left(\neg \alpha_{i}\right)\right)$ contains, in general, too few worlds (or, from another point of view, why the set $B_{\mathbb{S}, 1}$ contains, in general, too few sentences) for the operation $\div_{\mathbb{S}}^{3}$, defined in the previous subsection, to be a partial meet multiple contraction.

We may observe from its definition, that the set $B_{\mathbb{S}, 1}$ is, roughly speaking, obtained from $B$ by discarding from it all the sentences $\alpha_{r}$ for which there is some other sentence $\alpha_{s}$ in $B$ such that $f_{\mathbb{S}}\left(\neg \alpha_{s}\right) \subset\left\|\neg \alpha_{r}\right\|$ and $f_{\mathbb{S}}\left(\neg \alpha_{s}\right) \neq$ $f_{\mathbb{S}}\left(\neg \alpha_{r}\right)$. However, as we have seen in Counterexample 3.3, for some sets $B$, such definition of $B_{\mathbb{S}, 1}$ leads to a situation in which for some $\alpha_{t} \in B \backslash B_{\mathbb{S}, 1}$ it holds that $\left(\|\mathbf{K}\| \cup\left(\bigcup_{\alpha_{i} \in B_{\mathbb{S}, 1}} f_{\mathbb{S}}\left(\neg \alpha_{i}\right)\right)\right) \cap\left\|\neg \alpha_{t}\right\|=\emptyset$ (and, therefore, $\alpha_{t} \in \mathbf{K} \div \div_{\mathbb{S}}^{3}$ $B$, which yields that, in that case, $\div_{\mathbb{S}}^{3}$ is not a partial meet multiple contraction on $\mathbf{K}$ ). Indeed, as it can be seen in the mentioned counterexample (where we have shown that $\alpha_{1} \in B$ but $\left.\left(\|\mathbf{K}\| \cup\left(\bigcup_{\alpha_{i} \in B_{\mathbb{S}, 1}} f_{\mathbb{S}}\left(\neg \alpha_{i}\right)\right)\right) \cap\left\|\neg \alpha_{1}\right\|=\emptyset\right)$, such a situation occurs whenever $\alpha_{t} \in B \backslash B_{\mathbb{S}, 1}$ is such that (i) $\alpha_{t} \in B \cap K$, (ii) there is only one sentence in $B$, say $\alpha_{q}$, which is, roughly speaking, responsible for the fact that $\alpha_{t} \notin B_{\mathbb{S}, 1}$, (iii) for all $\alpha_{p} \in B \backslash\left\{\alpha_{t}, \alpha_{q}\right\}$ it holds that $f_{\mathbb{S}}\left(\neg \alpha_{p}\right) \cap\left\|\neg \alpha_{t}\right\|=$ $\emptyset$ and (iv) $\alpha_{q} \notin B_{\mathbb{S}, 1}$.

Hence, having the above in mind, it seems that, for any given set $B$ not containing tautologies, the subset $B_{\mathbb{S}}$ of $B$ that we wish to define should be constructed in a way that, if $\alpha_{r}, \alpha_{s} \in B \cap \mathbf{K}$ are two sentences in the above described conditions then they do not simultaneously belong to $B_{\mathbb{S}}$ but, on the other hand, $B_{\mathbb{S}}$ must be such that for all sentences $\alpha_{l} \in(B \cap \mathbf{K}) \backslash B_{\mathbb{S}}$ there is some $\alpha_{k} \in B_{\mathbb{S}}$ such that $f_{\mathbb{S}}\left(\neg \alpha_{k}\right) \subset\left\|\neg \alpha_{l}\right\|$ and $f_{\mathbb{S}}\left(\neg \alpha_{k}\right) \neq f_{\mathbb{S}}\left(\neg \alpha_{l}\right)$. The following theorem asserts that the above analysis is correct:

Theorem 3.4 Let $\mathbf{K}$ be a belief set and $\mathbb{S}$ be a system of spheres centred on $\|\mathbf{K}\|$. Let $\div$ s be an operation defined on $\mathbf{K}$ by:

$$
\mathbf{K} \div{ }_{\mathbb{S}} B= \begin{cases}T h\left(\|\mathbf{K}\| \cup\left(\bigcup_{\alpha_{i} \in B_{\mathbb{S}}} f_{\mathbb{S}}\left(\neg \alpha_{i}\right)\right)\right) & \text { if } B \cap C n(\emptyset)=\emptyset \\ \mathbf{K} & \text { if } B \cap C n(\emptyset) \neq \emptyset\end{cases}
$$


where, for any finite set of sentences $B$ satisfying $B \cap C n(\emptyset)=\emptyset$, the set $B_{\mathbb{S}}$ is a subset of $B$ such that the following conditions hold:

(i) If $\alpha_{j} \in B_{\mathbb{S}}$ then $f_{\mathbb{S}}\left(\neg \alpha_{k}\right)=f_{\mathbb{S}}\left(\neg \alpha_{j}\right)$ or $f_{\mathbb{S}}\left(\neg \alpha_{k}\right) \not \subset\left\|\neg \alpha_{j}\right\|$, for all $\alpha_{k} \in B_{\mathbb{S}}$.

(ii) For all $\alpha_{l} \in B \cap \mathbf{K}$ there is some $\alpha_{m} \in B_{\mathbb{S}} \cap \mathbf{K}$ such that $f_{\mathbb{S}}\left(\neg \alpha_{m}\right) \subseteq\left\|\neg \alpha_{l}\right\|$.

(iii) For any finite set of sentences $C$ satisfying $C \cap C n(\emptyset)=\emptyset$, if every subset $X$ of $\mathbf{K}$ implies some element of $B$ if and only if $X$ implies some element of $C$, then for all $\beta_{i} \in\left(B_{N} \cap \mathbf{K}\right)_{\mathbb{S}}$ there is some $\varsigma_{i} \in\left(C_{N} \cap \mathbf{K}\right)_{\mathbb{S}}$ such that $\left\|\beta_{i}\right\|=\left\|\varsigma_{i}\right\|$.

(iv) $B_{\mathbb{S}} \cap \mathbf{K}=(B \cap \mathbf{K})_{\mathbb{S}}$.

(v) $\left(B_{N}\right)_{\mathbb{S}}=\left(B_{\mathbb{S}}\right)_{N}$.

(vi) $\bigcup_{\alpha_{i} \in B_{\mathbb{S}}} f_{\mathbb{S}}\left(\neg \alpha_{i}\right)=\bigcup_{\alpha_{i} \in\left(B_{\mathbb{S}}\right)_{N}} f_{\mathbb{S}}\left(\neg \alpha_{i}\right)$.

Then the operation $\div \mathbb{s}$ is a partial meet multiple contraction on $\mathbf{K}$.

Conditions (i) and (ii) in the statement of the above theorem are motivated by our previous analysis. It is also worth noticing that the second one of those conditions assures that the set $\bigcup_{\alpha_{i} \in B_{\mathbb{S}}} f_{\mathbb{S}}\left(\neg \alpha_{i}\right)$ contains some $\neg \alpha_{l}$-world for all sentences $\alpha_{l} \in B \cap \mathbf{K}$ and, consequently, the operation $\div \mathbb{S}$ defined as suggested in the statement of the above theorem is such that the set $\mathbf{K} \div \mathbb{S} B$ does not contain any elements from $B$. Conditions (iv) - (vi) are technical constraints necessary for the proof.

\subsection{Spheres' System-based Multiple Contractions}

In this subsection we will start by presenting a way of systematically obtaining, for any finite set of sentences $B$, a subset $B_{\mathbb{S}}$ such that, whenever $B \cap C n(\emptyset)=$ $\emptyset$, the conditions (i) - (vi) stated in Theorem 3.4 are satisfied. After that we will make use of the constructed sets $B_{\mathbb{S}}$ to present a definition for the S.S.-based multiple contractions.

At this point we start by remarking that condition (i) of the mentioned theorem presents in some sense a necessary condition for an element $\alpha_{j}$ of $B$ to belong to $B_{\mathbb{S}}$ (namely: for all $\alpha_{k} \in B_{\mathbb{S}}$ it holds that $f_{\mathbb{S}}\left(\neg \alpha_{k}\right)=f_{\mathbb{S}}\left(\neg \alpha_{j}\right)$ or $\left.f_{\mathbb{S}}\left(\neg \alpha_{k}\right) \not \subset\left\|\neg \alpha_{j}\right\|\right)$. We will use that condition as our guideline towards the definition of a set $B_{\mathbb{S}}$ in the desired conditions. Taking this into account and observing that the condition imposed to be satisfied by all elements of $B_{\mathbb{S}}$ is recurring (in the sense that it makes reference to the set $B_{\mathbb{S}}$ itself) we are led to believe that the definition of $B_{\mathbb{S}}$ shall follow an iterative procedure.

Having this in mind we suggest the following construction for the set $B_{\mathbb{S}}: 5$

Definition 3.5 Let $\mathbf{K}$ be a belief set and $\mathbb{S}$ be a system of spheres centred on $\|\mathbf{K}\|$.

Consider a set of sentences $B=\left\{\alpha_{1}, \ldots, \alpha_{n}\right\} \subseteq \mathcal{L}$ such that $B \backslash C n(\emptyset) \neq \emptyset$.

\footnotetext{
${ }^{5}$ Further bellow we provide a more detailed explanation of the intuition behind this definition.
} 
Denote by $C_{1}, \ldots, C_{m}$ the (different) equivalence classes in the quotient set of $(B \backslash C n(\emptyset))$ by $\backsim$, i.e. $\left\{C_{1}, \ldots, C_{m}\right\}=(B \backslash C n(\emptyset)) / \backsim$, where $\backsim$ is the equivalence relation on $B \backslash C n(\emptyset)$ defined by:

$$
\forall \alpha, \beta \in B \backslash C n(\emptyset), \alpha \backsim \beta \text { iff } \mathbb{S}_{\neg \alpha}=\mathbb{S}_{\neg \beta},
$$

Moreover, assume that the equivalence classes $C_{1}, \ldots, C_{m}$ are ordered according the following condition:

$$
\text { If } 1 \leq i<j \leq m \text { then } \forall \alpha_{r} \in C_{i} \forall \alpha_{s} \in C_{j} \mathbb{S}_{\neg \alpha_{s}} \subset \mathbb{S}_{\neg \alpha_{r}} \text {. }
$$

Now consider the following list of subsets of $B$ :

$$
\begin{aligned}
& B_{0}=B \cap C n(\emptyset) \\
& C_{1}^{\prime}=C_{1} \\
& C_{1}^{\prime \prime}=\left\{\alpha_{i} \in C_{1}^{\prime}: \forall \alpha_{j} \in C_{1}^{\prime} f_{\mathbb{S}}\left(\neg \alpha_{j}\right) \not \subset f_{\mathbb{S}}\left(\neg \alpha_{i}\right)\right\} \\
& B_{1}=C_{1}^{\prime \prime}
\end{aligned}
$$

Moreover, if $m>1$ for all $l \in\{2, \ldots, m\}$, let $C_{l}^{\prime}, C_{l}^{\prime \prime}$ and $B_{l}$ be the sets defined by:

$$
\begin{aligned}
& C_{l}^{\prime}=\left\{\alpha_{i} \in C_{l}: \forall \alpha_{j} \in B_{l-1} f_{\mathbb{S}}\left(\neg \alpha_{j}\right) \not \subset\left\|\neg \alpha_{i}\right\|\right\} ; \\
& C_{l}^{\prime \prime}=\left\{\alpha_{i} \in C_{l}^{\prime}: \forall \alpha_{j} \in C_{l}^{\prime} f_{\mathbb{S}}\left(\neg \alpha_{j}\right) \not \subset f_{\mathbb{S}}\left(\neg \alpha_{i}\right)\right\} ; \\
& B_{l}=B_{l-1} \cup C_{l}^{\prime \prime} .
\end{aligned}
$$

The set $B_{\mathbb{S}}=B_{m}$ is the $\mathbb{S}$-based filtration of $B$.

If $D$ is a set of sentences such that $D \subseteq \operatorname{Cn}(\emptyset)$, then the $\mathbb{S}$-based filtration of $D$ is the empty set and is denoted by $D_{\mathbb{S}}$, i.e. $D_{\mathbb{S}}=\emptyset$.

The following observation presents an alternative (equivalent) way of defining the sets $C_{1}, \ldots, C_{m}, C_{1}^{\prime}, \ldots, C_{m}^{\prime}, C_{1}^{\prime \prime}, \ldots, C_{m}^{\prime \prime}$ introduced in the above definition.

Observation 3.6 Let $\mathbf{K}$ be a belief set, $\mathbb{S}$ be a system of spheres centred on $\|\mathbf{K}\|$ and $B$ be a finite set of sentences such that $B \backslash C n(\emptyset) \neq \emptyset$. Furthermore, let $C_{1}, \ldots, C_{m}, C_{1}^{\prime}, \ldots, C_{m}^{\prime}, C_{1}^{\prime \prime}, \ldots, C_{m}^{\prime \prime}, B_{1}, \ldots, B_{m}$ be the subsets of $B$ constructed as we described in the above definition. Then:

(a) For all $l \in\{1, \ldots, m\}$ it holds that $C_{l}^{\prime \prime}=\left\{\alpha_{i} \in C_{l}^{\prime}: \forall \alpha_{j} \in C_{l}^{\prime}\left(f_{\mathbb{S}}\left(\neg \alpha_{j}\right)=\right.\right.$ $f_{\mathbb{S}}\left(\neg \alpha_{i}\right)$ or $\left.\left.f_{\mathbb{S}}\left(\neg \alpha_{j}\right) \not \subset\left\|\neg \alpha_{i}\right\|\right)\right\}$.

(b) If $m>1$ then for all $l \in\{2, \ldots, m\}$ the following identity is satisfied: $C_{l}^{\prime}=$ $\left\{\alpha_{i} \in C_{l}: \forall \alpha_{j} \in B_{l-1}\left(f_{\mathbb{S}}\left(\neg \alpha_{j}\right)=f_{\mathbb{S}}\left(\neg \alpha_{i}\right)\right.\right.$ or $\left.\left.f_{\mathbb{S}}\left(\neg \alpha_{j}\right) \not \subset\left\|\neg \alpha_{i}\right\|\right)\right\}$.

We are now in a position to expose the intuition behind the above definition of the $\mathbb{S}$-based filtration of $B$. In order to do that we must start by remarking that our intention when proposing the above definition was that the set $B_{\mathbb{S}} \subseteq B$ should be such that, whenever $B$ is a set that does not contain any tautology, conditions (i) and (ii) of Theorem 3.4 are satisfied. 
If we were only interested in the fulfilment of condition (i) above, then it would be enough to define $B_{\mathbb{S}}=\left\{\alpha_{i} \in B: \forall \alpha_{k} \in B \backslash \operatorname{Cn}(\emptyset)\left(f_{\mathbb{S}}\left(\neg \alpha_{k}\right)=\right.\right.$ $f_{\mathbb{S}}\left(\neg \alpha_{i}\right)$ or $\left.\left.f_{\mathbb{S}}\left(\neg \alpha_{k}\right) \not \subset\left\|\neg \alpha_{i}\right\|\right)\right\}\left(=B_{\mathbb{S}, 1}\right)$, however, as we have concluded with the help of Counterexample 3.3, if $B_{\mathbb{S}}$ was defined in that way then condition (ii) would not, in general, be satisfied.

Recalling the situation described in Counterexample 3.3 we can observe that, in those circumstances, condition (ii) is not satisfied essentially because, among other facts, it holds that $f_{\mathbb{S}}\left(\neg \alpha_{2}\right) \subset\left\|\neg \alpha_{1}\right\|$ and $f_{\mathbb{S}}\left(\neg \alpha_{3}\right) \subset\left\|\neg \alpha_{2}\right\|$, while, on the other hand, $f_{\mathbb{S}}\left(\neg \alpha_{3}\right) \nsubseteq\left\|\neg \alpha_{1}\right\|$. Roughly speaking, in those conditions, what happens is that the sentence $\alpha_{2} \in B$, which is responsible for the noninclusion of $\alpha_{1}$ in $B_{\mathbb{S}}$, does not belong to the set $B_{\mathbb{S}}$ either, and that fact (together with the remaining circumstances) causes that for all $\alpha_{m} \in B_{\mathbb{S}} \cap \mathbf{K}$ it holds that $f_{\mathbb{S}}\left(\neg \alpha_{m}\right) \nsubseteq\left\|\neg \alpha_{l}\right\|$.

Now notice that, given two non tautological sentences $\alpha$ and $\beta$, it follows immediately from Definition 2.2 that if $f_{\mathbb{S}}(\neg \alpha) \subset\|\neg \beta\|$ then $\mathbb{S}_{\neg \beta} \subseteq \mathbb{S}_{\neg \alpha}$. On the other hand, if $\mathbb{S}_{\neg \alpha}=\mathbb{S}_{\neg \beta}$ and $f_{\mathbb{S}}(\neg \alpha) \subset\|\neg \beta\|$ then $f_{\mathbb{S}}(\neg \alpha) \subseteq f_{\mathbb{S}}(\neg \beta)$.

Hence, we can conclude that conditions $f_{\mathbb{S}}\left(\neg \alpha_{q}\right) \subset\left\|\neg \alpha_{p}\right\|, f_{\mathbb{S}}\left(\neg \alpha_{r}\right) \subset\left\|\neg \alpha_{q}\right\|$ and $f_{\mathbb{S}}\left(\neg \alpha_{r}\right) \nsubseteq\left\|\neg \alpha_{p}\right\|$ can only hold simultaneously as long as $\mathbb{S}_{\neg \alpha_{q}} \subset \mathbb{S}_{\neg \alpha_{r}}$.

Therefore, from all the above we can observe that:

1. In order to assure that the set $B_{\mathbb{S}} \subseteq B$ satisfies conditions (i)-(ii) mentioned above, then it must be defined in a way which imposes that a sentence $\alpha_{i} \in B$ only belongs to $B_{\mathbb{S}}$ if $f_{\mathbb{S}}\left(\neg \alpha_{k}\right)=f_{\mathbb{S}}\left(\neg \alpha_{i}\right)$ or $f_{\mathbb{S}}\left(\neg \alpha_{k}\right) \not \subset$ $\left\|\neg \alpha_{i}\right\|$, for all sentences $\alpha_{k}$ which are, themselves, elements of $B_{\mathbb{S}}$ (but not necessarily for all sentences of $B$ ).

2. If $C$ is a finite set such that $C \cap C n(\emptyset)=\emptyset$ and for some $\mathcal{D} \in \mathbb{S}$ it holds that $\mathbb{S}_{\neg \alpha_{i}}=\mathcal{D}$, for all $\alpha_{i} \in C \cap \mathbf{K}$, and $C_{\mathbb{S}}=\left\{\alpha_{i} \in C: \forall \alpha_{k} \in C\left(f_{\mathbb{S}}\left(\neg \alpha_{k}\right)=\right.\right.$ $f_{\mathbb{S}}\left(\neg \alpha_{i}\right)$ or $\left.\left.f_{\mathbb{S}}\left(\neg \alpha_{k}\right) \not \subset\left\|\neg \alpha_{i}\right\|\right)\right\}$ then the conditions obtained from (i) and (ii), by replacing, in each of those clauses, $B$ by $C$, are satisfied. ${ }^{6}$

3. If $\alpha$ and $\beta$ are two sentences such that $\mathbb{S}_{\neg \alpha} \subset \mathbb{S}_{\neg \beta}$ then $f_{\mathbb{S}}(\neg \alpha) \not \subset\|\neg \beta\|$.

Since it is based in the facts listed above, we are now in a position to explain the idea behind the definition of the $\mathbb{S}$-based filtration of a set $B$ (Definition 3.5). For that purpose in what follows we assume that $B$ is a finite set such that $B \backslash C n(\emptyset) \neq \varnothing$ and describe how the set $B_{\mathbb{S}}$ is obtained as well as the motivations behind such procedure.

The first concern underlying such definition has to do with point 1 . above and consists in constructing the set $B_{\mathbb{S}}$ a the result of a sequence of steps (rather than by a single step definition) in order to assure that at each stage of that construction we only prevent from being included in $B_{\mathbb{S}}$ those sentences $\alpha_{i} \in B$ such that $f_{\mathbb{S}}\left(\neg \alpha_{k}\right) \neq f_{\mathbb{S}}\left(\neg \alpha_{i}\right)$ and $f_{\mathbb{S}}\left(\neg \alpha_{k}\right) \subset\left\|\neg \alpha_{i}\right\|$, for some sentence $\alpha_{k} \in B$ which is somehow guaranteed to be such that it will belong to the set $B_{\mathbb{S}}$ when its ongoing construction is finished.

\footnotetext{
${ }^{6}$ Notice that a rigorous proof that this indeed holds can be trivially obtained by combining Observation 3.6 and Lemma A.4.
} 
So, as it can be seen from its definition above, in order to construct the set $B_{\mathbb{S}}$ we start by partitioning $B$ in several classes $B_{0}, C_{1}, \ldots, C_{m}$, where $B_{0}=$ $B \cap C n(\emptyset)$ and for each $i \in\{1, \ldots, m\}$ the class $C_{i}$ is such that if $\alpha_{r} \in C_{i}$ then for all $\alpha_{s} \in B \backslash C n(\emptyset)$ it holds that $\alpha_{s} \in C_{i}$ if and only if $\mathbb{S}_{\neg \alpha_{s}}=\mathbb{S}_{\neg \alpha_{r}}$ (hence $m \geq 1$ is uniquely determined by the set $B$ and the system of spheres $\mathbb{S}$ ). Furthermore, given two such classes $C_{i}$ and $C_{j}$, if $i<j, \alpha_{r} \in C_{i}$ and $\alpha_{s} \in C_{j}$ then $\mathbb{S}_{\neg \alpha_{s}} \subset \mathbb{S}_{\neg \alpha_{r}}$.

In the above conditions we have that, for each $i \in\{1, \ldots, m\}$ the class $C_{i}$ is such that if $\alpha_{r} \in C_{i}$ and $i<j$, with $j \in\{1, \ldots, m\}$, then for all $\alpha_{s} \in C_{j}$ it holds that $f_{\mathbb{S}}\left(\neg \alpha_{s}\right) \not \subset\left\|\alpha_{r}\right\|$. Hence, if $\alpha_{p} \in C_{1}$ then $f_{\mathbb{S}}\left(\neg \alpha_{q}\right)=f_{\mathbb{S}}\left(\neg \alpha_{p}\right)$ or $f_{\mathbb{S}}\left(\neg \alpha_{q}\right) \not \subset$ $\left\|\neg \alpha_{p}\right\|$, for all $\alpha_{q} \in B \backslash\left(B_{0} \cup C_{1}\right)$. However, there can be some sentences $\alpha_{l} \in$ $C_{1}$ for which it does not hold that $f_{\mathbb{S}}\left(\neg \alpha_{m}\right)=f_{\mathbb{S}}\left(\neg \alpha_{l}\right)$ or $f_{\mathbb{S}}\left(\neg \alpha_{m}\right) \not \subset\left\|\neg \alpha_{l}\right\|$, for all $\alpha_{m} \in C_{1}$.

So, naturally, the next step in the construction of the set $B_{\mathbb{S}}$ consists in obtaining the subset of $C_{1}$ defined by $C_{1}^{\prime \prime}=\left\{\alpha_{i} \in C_{1}^{\prime}: \forall \alpha_{j} \in C_{1}^{\prime} f_{\mathbb{S}}\left(\neg \alpha_{j}\right) \not \subset\right.$ $\left.f_{\mathbb{S}}\left(\neg \alpha_{i}\right)\right\}$, where $C_{1}^{\prime}=C_{1}{ }^{7}$ It follows immediately from its construction and some of our remarks above that the set $C_{1}^{\prime \prime}$ is such that, on the one hand, if $\alpha_{p} \in C_{1}^{\prime \prime}$ then $f_{\mathbb{S}}\left(\neg \alpha_{q}\right)=f_{\mathbb{S}}\left(\neg \alpha_{p}\right)$ or $f_{\mathbb{S}}\left(\neg \alpha_{q}\right) \not \subset\left\|\neg \alpha_{p}\right\|$, for all $\alpha_{q} \in C_{1}^{\prime \prime} \cup(B \backslash$ $\left.\left(B_{0} \cup C_{1}\right)\right)$ and, on the other hand, for all $\alpha_{l} \in C_{1} \backslash C_{1}^{\prime \prime}$ there is some $\alpha_{m} \in C_{1}^{\prime \prime}$ such that $f_{\mathbb{S}}\left(\neg \alpha_{m}\right) \neq f_{\mathbb{S}}\left(\neg \alpha_{l}\right)$ and $f_{\mathbb{S}}\left(\neg \alpha_{m}\right) \subset\left\|\neg \alpha_{l}\right\|$ (see footnote 6). At this point we define the set $B_{1}=C_{1}^{\prime \prime}$, which is the set of sentences of $C_{1}$ that we will include in the set $B_{\mathbb{S}}$. Indeed, if $B \backslash\left(B_{0} \cup C_{1}\right)=\emptyset$ (or, equivalently, if $m=1$ ) then the set $B_{\mathbb{S}}$ is simply defined by $B_{\mathbb{S}}=B_{1}$.

If, instead, $B \backslash\left(B_{0} \cup C_{1}\right) \neq \varnothing$ (or, equivalently, if $\left.m>1\right)$, the next phase consists in choosing which sentences of $C_{2}$ shall be included in the set $B_{\mathbb{S}}$. We do this in two steps. First we consider the set $C_{2}^{\prime}=\left\{\alpha_{i} \in C_{2}: \forall \alpha_{j} \in\right.$ $\left.B_{1} f_{\mathbb{S}}\left(\neg \alpha_{j}\right) \not \subset\left\|\neg \alpha_{i}\right\|\right\}^{8}$ which consists of the subset of $C_{2}$ such that $\alpha_{i} \in C_{2}^{\prime}$ if and only if $\alpha_{i} \in C_{2}$ and $f_{\mathbb{S}}\left(\neg \alpha_{j}\right) \not \subset\left\|\neg \alpha_{i}\right\|$ (or $f_{\mathbb{S}}\left(\neg \alpha_{j}\right)=f_{\mathbb{S}}\left(\neg \alpha_{i}\right)$ ), for all $\alpha_{j} \in$ $B_{1} \cup\left(B \backslash\left(B_{0} \cup C_{1} \cup C_{2}\right)\right)$. Moreover, we also have that if $\alpha_{l} \in C_{2} \backslash C_{2}^{\prime}$ then there is some $\alpha_{m} \in B_{1}$ such that $f_{\mathbb{S}}\left(\neg \alpha_{m}\right) \neq f_{\mathbb{S}}\left(\neg \alpha_{l}\right)$ and $f_{\mathbb{S}}\left(\neg \alpha_{m}\right) \subset\left\|\neg \alpha_{l}\right\|$. However there may be some sentence $\alpha_{k} \in C_{2}^{\prime}$ such that $f_{\mathbb{S}}\left(\neg \alpha_{h}\right) \subset\left\|\neg \alpha_{k}\right\|$ and $f_{\mathbb{S}}\left(\neg \alpha_{h}\right) \neq f_{\mathbb{S}}\left(\neg \alpha_{k}\right)$ for some $\alpha_{h} \in C_{2}^{\prime}$. Hence, secondly we must obtain the subset $C_{2}^{\prime \prime} \subseteq C_{2}^{\prime}$ which represents to $C_{2}^{\prime}$ the same that the set $C_{1}^{\prime \prime}$ represented to $C_{1}^{\prime \prime}$, i.e. $C_{2}^{\prime \prime}=\left\{\alpha_{i} \in C_{2}^{\prime}: \forall \alpha_{j} \in C_{2}^{\prime} f_{\mathbb{S}}\left(\neg \alpha_{j}\right) \not \subset f_{\mathbb{S}}\left(\neg \alpha_{i}\right)\right\}$. Afterwards we define $B_{2}=B_{1} \cup C_{2}^{\prime \prime}$. Therefore $B_{2}$ is a subset of $C_{1} \cup C_{2}$ such that (a) if $\alpha_{i} \in B_{2}$ then $f_{\mathbb{S}}\left(\neg \alpha_{j}\right)=f_{\mathbb{S}}\left(\neg \alpha_{i}\right)$ or $f_{\mathbb{S}}\left(\neg \alpha_{j}\right) \not \subset\left\|\neg \alpha_{i}\right\|$, for all $\alpha_{j} \in B_{2} \cup\left(B \backslash\left(B_{0} \cup C_{1} \cup C_{2}\right)\right)$ and (b) for all $\alpha_{l} \in\left(C_{1} \cup C_{2}\right) \backslash B_{2}$ there is some $\alpha_{m} \in B_{2}$ such that $f_{\mathbb{S}}\left(\neg \alpha_{m}\right) \neq$ $f_{\mathbb{S}}\left(\neg \alpha_{l}\right)$ and $f_{\mathbb{S}}\left(\neg \alpha_{m}\right) \subset\left\|\neg \alpha_{l}\right\|$. Now, if $B \backslash\left(B_{0} \cup C_{1} \cup C_{2}\right)=\emptyset$ (or, equivalently, if $m=2$ ) then we just define $B_{\mathbb{S}}=B_{2}$. Otherwise, we obtain the set $B_{3}$ by applying to the class $C_{3}$ the same procedure (with the necessary obvious adaptations) that we have just described above regarding the class $C_{2}$, and we

\footnotetext{
${ }^{7}$ Notice that, according to Observation 3.6, $C_{1}^{\prime \prime}=\left\{\alpha_{i} \in C_{1}^{\prime}: \forall \alpha_{j} \in C_{1}^{\prime}\left(f_{\mathbb{S}}\left(\neg \alpha_{j}\right)=f_{\mathbb{S}}\left(\neg \alpha_{i}\right)\right.\right.$ or $\left.\left.f_{\mathbb{S}}\left(\neg \alpha_{j}\right) \not \subset\left\|\neg \alpha_{i}\right\|\right)\right\}$.

${ }^{8}$ Notice that, according to Observation 3.6, $C_{2}^{\prime}=\left\{\alpha_{i} \in C_{2}: \forall \alpha_{j} \in B_{1}\left(f_{\mathbb{S}}\left(\neg \alpha_{j}\right)=f_{\mathbb{S}}\left(\neg \alpha_{i}\right)\right.\right.$ or $\left.\left.f_{\mathbb{S}}\left(\neg \alpha_{j}\right) \not \subset\left\|\neg \alpha_{i}\right\|\right)\right\}$.
} 
go on repeating this process until we have obtained the set $B_{m}$. Then, finally, we define $B_{\mathbb{S}}=B_{m}$ and we are done.

The following observation asserts that the $\mathbb{S}$-based filtration of $B$ satisfies the conditions (i) $-($ vi) of Theorem 3.4.

Observation 3.7 Let $\mathbf{K}$ be a belief set, $\mathbb{S}$ be a system of spheres centred on $\|\mathbf{K}\|$ and $B$ be a set of sentences such that $B \cap C n(\emptyset)=\emptyset$. If $B_{\mathbb{S}}$ is the $\mathbb{S}$-based filtration of $B$, then:

(i) If $\alpha_{j} \in B_{\mathbb{S}}$ then $f_{\mathbb{S}}\left(\neg \alpha_{i}\right)=f_{\mathbb{S}}\left(\neg \alpha_{j}\right)$ or $f_{\mathbb{S}}\left(\neg \alpha_{i}\right) \not \subset\left\|\neg \alpha_{j}\right\|$, for all $\alpha_{i} \in B_{\mathbb{S}}$.

(ii) For all $\alpha_{l} \in B \cap \mathbf{K}$ there is some $\alpha_{k} \in B_{\mathbb{S}} \cap \mathbf{K}$ such that $f_{\mathbb{S}}\left(\neg \alpha_{k}\right) \subseteq\left\|\neg \alpha_{l}\right\|$.

(iii) For any set of sentences $C$, if every subset $X$ of $\mathbf{K}$ implies some element of $B$ if and only if $X$ implies some element of $C$, then for all $\beta_{i} \in\left(B_{N} \cap \mathbf{K}\right)_{\mathbb{S}}$ there is some $\varsigma_{i} \in\left(C_{N} \cap \mathbf{K}\right)_{\mathbb{S}}$ such that $\left\|\beta_{i}\right\|=\left\|\varsigma_{i}\right\|$.

(iv) $B_{\mathbb{S}} \cap \mathbf{K}=(B \cap \mathbf{K})_{\mathbb{S}}$.

(v) $\left(B_{N}\right)_{\mathbb{S}}=\left(B_{\mathbb{S}}\right)_{N}$.

(vi) $\bigcup_{\alpha_{i} \in B_{\mathbb{S}}} f_{\mathbb{S}}\left(\neg \alpha_{i}\right)=\bigcup_{\alpha_{i} \in\left(B_{\mathbb{S}}\right)_{N}} f_{\mathbb{S}}\left(\neg \alpha_{i}\right)$.

Now, having in mind Theorem 3.4 and the above observation, we are naturally led to suggest the following definition for the system of spheres-based multiple contractions:

Definition 3.8 (System of Spheres-based Multiple Contractions)

Let $\mathbf{K}$ be a belief set and $\mathbb{S}$ be a system of spheres centred on $\|\mathbf{K}\|$. The $\mathbb{S}$-based multiple contraction on $\mathbf{K}$ is the multiple contraction function $\div \mathbb{S}$ defined by:

$$
\mathbf{K} \div{ }_{\mathbb{S}} B= \begin{cases}T h\left(\|\mathbf{K}\| \cup\left(\bigcup_{\alpha_{i} \in B_{\mathbb{S}}} f_{\mathbb{S}}\left(\neg \alpha_{i}\right)\right)\right) & , \text { if } B \cap C n(\emptyset)=\varnothing \\ \mathbf{K} & \text {, if } B \cap C n(\emptyset) \neq \varnothing\end{cases}
$$

for any set of sentences $B$ and where $B_{\mathbb{S}}$ is the $\mathbb{S}$-based filtration of $B$. An operator $\div$ on $\mathbf{K}$ is a system of spheres-based multiple contraction on $\mathbf{K}$ if and only if there is a system of spheres $\mathbb{S}$ centred on $\|\mathbf{K}\|$, such that $\mathbf{K} \div B=\mathbf{K} \div{ }_{\mathbb{S}} B$, for any set of sentences $B$.

We are finally in a position to present the following main result of the present paper which confirms that every system of spheres-based multiple contraction is a partial meet multiple contraction.

Theorem 3.9 Let $\mathbf{K}$ be a belief set and $\mathbb{S}$ be a system of spheres centred on $\|\mathbf{K}\|$. Then the $\mathbb{S}$-based multiple contraction on $\mathbf{K}$ is a partial meet multiple contraction on $\mathbf{K}$.

The next observation shows that the above defined system of spheres-based multiple contractions are indeed a generalization of Grove's system of spheresbased (singleton) contractions. 
Fig. 4 Illustration of Example 3.11

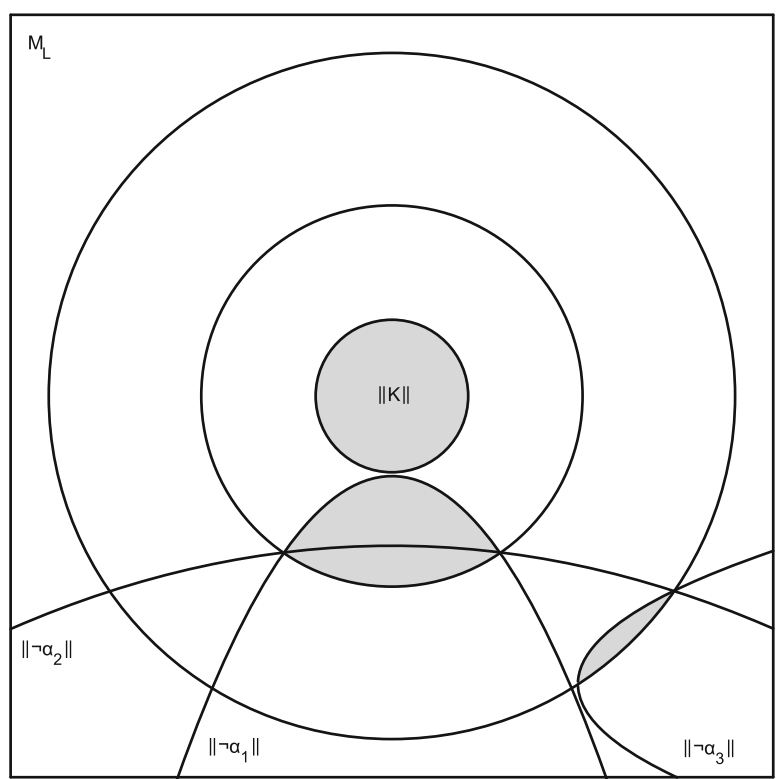

Observation 3.10 Let $\mathbf{K}$ be a belief set and $\mathbb{S}$ be a system of spheres centred on $\|\mathbf{K}\|$. If $\div \mathbb{S}$ is the $\mathbb{S}$-based multiple contraction and $-_{\mathbb{S}}$ is the $\mathbb{S}$-based (singleton) contraction, then:

1. For any set of sentences $B$ such that $B \cap C n(\emptyset)=\emptyset$ it holds that:

$$
\mathbf{K} \div \mathbb{S} B=\bigcap_{\alpha_{i} \in B_{\mathbb{S}}} \mathbf{K} \div \mathbb{S}\left\{\alpha_{i}\right\}=\bigcap_{\alpha_{i} \in B_{\mathbb{S}}} \mathbf{K}-\mathbb{S} \alpha_{i},
$$

where $B_{\mathbb{S}}$ is the $\mathbb{S}$-based filtration of $B$.

2. The identity $\mathbf{K} \div{ }_{\mathbb{S}}\{\alpha\}=\mathbf{K}-\mathbb{s} \alpha$ is satisfied for any sentence $\alpha \in \mathcal{L}$.

\subsubsection{An Example of the Construction}

Now, with the goal of clarifying the idea behind Definitions 3.5 and 3.8, we present an example illustrating the obtention of a set $\mathbf{K} \div{ }_{\mathbb{S}} B$ :

Example 3.11 Consider Fig. 4. Let $\mathbf{K}$ be a belief set and $\alpha_{1}, \alpha_{2}$ and $\alpha_{3}$ be sentences such that the sets $\|\mathbf{K}\|,\left\|\neg \alpha_{1}\right\|,\left\|\neg \alpha_{2}\right\|$ and $\left\|\neg \alpha_{3}\right\|$ can be represented as in that figure. Furthermore, consider the set $B=\left\{\alpha_{1}, \alpha_{2}, \alpha_{3}\right\}$ and let $\mathbb{S}$ be the system of spheres centred on $\|\mathbf{K}\|$ whose spheres are $\mathcal{M}_{\mathcal{L}}$ and the sets of possible worlds represented by each of the circles present in the mentioned figure.

In what follows we obtain the $\mathbb{S}$-based filtration $B_{\mathbb{S}}$ of the set $B$ and we clarify how the set $\mathbf{K} \div \mathbb{S} B$ is obtained, where $\div \mathbb{S}$ is the $\mathbb{S}$-based multiple contraction on $\mathbf{K}$. So, according to the process of construction of $B_{\mathbb{S}}$ described 
in Definition 3.5 and based on the information present in the mentioned figure we obtain that:

- $\quad B_{0}=B \cap C n(\emptyset)=\emptyset$.

- $(B \backslash C n(\emptyset)) / \sim$ is composed of two equivalence classes (and, therefore, $m=2): C_{1}=\left\{\alpha_{3}\right\}$ and $C_{2}=\left\{\alpha_{1}, \alpha_{2}\right\}$.

- $B_{1}=C_{1}^{\prime \prime}=C_{1}^{\prime}=C_{1}=\left\{\alpha_{3}\right\}$

- $C_{2}^{\prime}=\left\{\alpha_{1}\right\}$ (notice that $\alpha_{2} \notin C_{2}^{\prime}$ because $f_{\mathbb{S}}\left(\neg \alpha_{3}\right) \subset\left\|\neg \alpha_{2}\right\|$ ).

- $C_{2}^{\prime \prime}=C_{2}^{\prime}=\left\{\alpha_{1}\right\}$.

- $\quad B_{\mathbb{S}}=B_{2}=B_{1} \cup C_{2}^{\prime \prime}=\left\{\alpha_{1}, \alpha_{3}\right\}$.

Hence, according to Definition 3.8, in the above conditions we have that $\mathbf{K} \div \mathbb{S} B=T h\left(\|\mathbf{K}\| \cup\left(\bigcup_{\alpha_{i} \in B_{\mathbb{S}}} f_{\mathbb{S}}\left(\neg \alpha_{i}\right)\right)\right)=T h\left(\|\mathbf{K}\| \cup f_{\mathbb{S}}\left(\neg \alpha_{1}\right) \cup f_{\mathbb{S}}\left(\neg \alpha_{3}\right)\right)$, that is, the set $\mathbf{K} \div \mathbb{S} B$ is given by the intersection of all the worlds in the shaded region of Fig. 4.

\section{Conclusions}

We have introduced a new class of multiple contraction functions-the S.S.-based multiple contractions - which are a generalization to the case of contraction by (possibly non-singleton) sets of sentences of Grove's S.S.based contraction functions. In particular we have shown that every S.S.-based multiple contraction is a partial meet multiple contraction.

In a paper which is currently in preparation we shall provide an axiomatic characterization for the newly introduced S.S.-based multiple contractions.

Having in mind that in the singleton contraction level the S.S.-based contractions are transitively relational partial meet contractions (in fact, those two classes of singleton contraction function are identical and constitute a subclass of the class of partial meet contractions), we are led to expect that the S.S.based multiple contraction functions that we have presented are transitively relational partial meet multiple contractions (TRPMMCs).

Nevertheless, in the present paper we have only focused in assuring that the new class of multiple contractions proposed was a subclass of the class of partial meet multiple contractions. We leave it as subject of future work to investigate if (as it is suggested by the analogy with the singleton case) it holds, additionally, that the S.S.-based multiple contractions are indeed TRPMMCs.

Acknowledgements We wish to thank the three referees of the JPL for their valuable comments on the previous version of the paper which have significantly contributed to improve its readability. 


\section{Appendix: Proofs}

Previous Lemmas

Lemma A.1 [10] Let $\mathbf{K}$ be a belief set and $B$ and $C$ be sets of sentences. Then the following statements hold:

(i) $B \cap C n(\emptyset) \neq \emptyset$ if and only if $\mathbb{W}_{\mathbf{K} \perp B}=\emptyset$.

(ii) $B \cap \mathbf{K}=\emptyset$ if and only if $\mathbb{W}_{\mathbf{K} \perp B}=\{\emptyset\}$.

(iii) $B \cap C n(\emptyset)=\emptyset$ and $B \cap \mathbf{K} \neq \emptyset$ if and only if $\mathbb{W}_{\mathbf{K} \perp B} \neq \emptyset$ and $\emptyset \notin \mathbb{W}_{\mathbf{K} \perp B}$.

(iv) $\mathbf{K} \perp B=\mathbf{K} \perp C$ iff $\mathbb{W}_{\mathbf{K} \perp B}=\mathbb{W}_{\mathbf{K} \perp C \text {. }}$

Lemma A.2 [10] Let $\mathbf{K}$ be a belief set and $B$ and $C$ be sets of sentences. Then:

(i) If $\alpha_{l} \in B$ and $\alpha_{l} \notin B_{N}$ then there is some $\alpha_{k} \in B_{N}$ such that $\left\|\neg \alpha_{k}\right\| \subset$ $\left\|\neg \alpha_{l}\right\|$.

(ii) If every subset $X$ of $\mathbf{K}$ implies some element of $B$ if and only if $X$ implies some element of $C$, then for all $\beta_{i} \in B_{N} \cap \mathbf{K}$ there is some $\varsigma_{i} \in C_{N} \cap \mathbf{K}$ such that $\left\|\beta_{i}\right\|=\left\|s_{i}\right\|$.

Lemma A.3 [10] Let $\mathbf{K}$ be a belief set, $B$ be a set of sentences such that $B \cap$ $C n(\emptyset)=\emptyset$ and $f:\{\|\alpha\|: \alpha \in \mathcal{L}$ and $\forall \neg \alpha\} \rightarrow \mathcal{P}\left(\mathcal{M}_{\mathcal{L}}\right)$ be a function such that if $\alpha \in \mathcal{L}$ and $\forall \neg \alpha$ then $\emptyset \neq f(\|\alpha\|) \subseteq\|\alpha\|$. If $B_{f} \subseteq B$ is such that:

(i) If $\alpha_{j} \in B_{f}$ then $f\left(\left\|\neg \alpha_{k}\right\|\right)=f\left(\left\|\neg \alpha_{j}\right\|\right)$ or $f\left(\left\|\neg \alpha_{k}\right\|\right) \not \subset\left\|\neg \alpha_{j}\right\|$, for all $\alpha_{k} \in$ $B_{f}$,

(ii) For all $\alpha_{l} \in B \cap \mathbf{K}$ there is some $\alpha_{m} \in B_{f} \cap \mathbf{K}$ such that $f\left(\left\|\neg \alpha_{m}\right\|\right) \subseteq$ $\left\|\neg \alpha_{l}\right\|$,

then $\left\{\mathcal{W} \in \mathbb{W}_{\mathbf{K} \perp B}: \mathcal{W} \subseteq \bigcup_{\alpha_{i} \in B_{f} \cap \mathbf{K}} f\left(\left\|\neg \alpha_{i}\right\|\right)\right\} \neq \emptyset$ and $\bigcup\left\{\mathcal{W} \in \mathbb{W}_{\mathbf{K} \perp B}: \mathcal{W} \subseteq\right.$ $\left.\bigcup_{\alpha_{i} \in B_{f} \cap \mathbf{K}} f\left(\left\|\neg \alpha_{i}\right\|\right)\right\}=\bigcup_{\alpha_{i} \in B_{f} \cap \mathbf{K}} f\left(\left\|\neg \alpha_{i}\right\|\right)$.

Lemma A.4 Let $\mathbf{K}$ be a belief set and $\mathbb{S}$ be a system of spheres centred on $\|\mathbf{K}\|$. Assume $C=\left\{\alpha_{1}, \ldots, \alpha_{n}\right\} \subseteq \mathcal{L} \backslash C n(\emptyset)$, with $n \geq 1$ is a non-empty finite set of sentences such that $\mathbb{S}_{\neg \alpha_{1}}=\cdots=\mathbb{S}_{\neg \alpha_{n}}$, and, for each $\alpha_{j} \in C$ let $C_{\alpha_{j}}$ be the set defined by $C_{\alpha_{j}}=\left\{\alpha_{k} \in C: f_{\mathbb{S}}\left(\neg \alpha_{k}\right) \subset f_{\mathbb{S}}\left(\neg \alpha_{j}\right)\right\}$.

If $\alpha_{j} \in C$ and $C_{\alpha_{j}} \neq \emptyset$ then there is some $\alpha_{l} \in C_{\alpha_{j}}$ such that $C_{\alpha_{l}}=\emptyset$.

Proof In what follows \#S denotes the number of elements of $S$.

Let $\alpha_{j} \in C$ be such that $C_{\alpha_{j}} \neq \emptyset$. It follows immediately from the definition of $C_{\alpha_{i}}$ that for any $\alpha_{r} \in C$, if $\alpha_{r} \in C_{\alpha_{j}}$ then $C_{\alpha_{r}} \subset C_{\alpha_{j}}$. Now we proceed according to the following (finite) sequence of steps:

Step 1: Pick some $\alpha_{j 1} \in C_{\alpha_{j}}$. Then $C_{\alpha_{j 1}} \subset C_{\alpha_{j}}$, hence $0 \leq \# C_{\alpha_{j 1}}<n-1$. 
Step 2: If $\# C_{\alpha_{j 1}}=0$ we have that $C_{\alpha_{j 1}}=\emptyset$ and then we can take $\alpha_{l}=\alpha_{j 1}$ and this finishes the proof. Otherwise, pick some $\alpha_{j 2} \in C_{\alpha_{j 1}}$. Then $C_{\alpha_{j 2}} \subset$ $C_{\alpha_{j 1}}$, hence $0 \leq \# C_{\alpha_{j 2}}<n-2$.

Step $i:$ If $\# C_{\alpha_{j(i-1)}}=0$ we have that $C_{\alpha_{j(i-1)}}=\emptyset$ and then we can take $\alpha_{l}=$ $\alpha_{j(i-1)}$ and this finishes the proof. Otherwise, pick some $\alpha_{j i} \in C_{\alpha_{j(i-1)}}$. Then $C_{\alpha_{j i}} \subset C_{\alpha_{j(i-1)}}$, hence $0 \leq \# C_{\alpha_{j i}}<n-i$.

Suppose after $n-2$ steps the above described process has not finished yet. Furthermore, assume that $\# C_{\alpha_{j(n-2)}} \neq 0$. Since, by construction, $0 \leq \# C_{\alpha_{j(n-2)}}<$ $n-(n-2)=2$, we can conclude that $C_{\alpha_{j(n-2)}}$ is a singleton set. Then, at step $n-1$ we pick the only element of $C_{\alpha_{j(n-2)}}$, which we denote by $\alpha_{j(n-1)}$, and from the fact that $C_{\alpha_{j(n-1)}} \subset C_{\alpha_{j(n-2)}}$ it must be the case that $C_{\alpha_{j(n-1)}}=\emptyset$. Hence, after at most $n$ steps the process must have finished. That is, at most at step $n$ we must find some $\alpha_{l} \in C_{\alpha_{j}}$ such that $C_{\alpha_{l}}=\emptyset$ as we wished to prove.

Lemma A.5 Let $\mathbf{K}$ be a belief set, $\mathbb{S}$ be a system of spheres centred on $\|\mathbf{K}\|$ and $B$ be a set of sentences such that $B \cap C n(\emptyset)=\emptyset$. If $B_{\mathbb{S}}$ is a subset of $B$ such that:

(i) If $\alpha_{j} \in B_{\mathbb{S}}$ then $f_{\mathbb{S}}\left(\neg \alpha_{k}\right)=f_{\mathbb{S}}\left(\neg \alpha_{j}\right)$ or $f_{\mathbb{S}}\left(\neg \alpha_{k}\right) \not \subset\left\|\neg \alpha_{j}\right\|$, for all $\alpha_{k} \in B_{\mathbb{S}}$.

(ii) For all $\alpha_{l} \in B \cap \mathbf{K}$ there is some $\alpha_{m} \in B_{\mathbb{S}} \cap \mathbf{K}$ such that $f_{\mathbb{S}}\left(\neg \alpha_{m}\right) \subseteq\left\|\neg \alpha_{l}\right\|$.

then $\left\{\mathcal{W} \in \mathbb{W}_{\mathbf{K} \perp B}: \mathcal{W} \subseteq \bigcup_{\alpha_{i} \in B_{\mathbb{S}} \cap \mathbf{K}} f_{\mathbb{S}}\left(\neg \alpha_{i}\right)\right\} \neq \emptyset$, and $\bigcup\left\{\mathcal{W} \in \mathbb{W}_{\mathbf{K} \perp B}: \mathcal{W} \subseteq\right.$ $\left.\bigcup_{\alpha_{i} \in B_{\mathbb{S}} \cap \mathbf{K}} f_{\mathbb{S}}\left(\neg \alpha_{i}\right)\right\}=\bigcup_{\alpha_{i} \in B_{\mathbb{S}} \cap \mathbf{K}} f_{\mathbb{S}}\left(\neg \alpha_{i}\right)$.

Proof Assume $B$ is a set of sentences satisfying $B \cap C n(\emptyset)=\emptyset$ and let $B_{\mathbb{S}}$ be a subset of $B$ such that conditions (i) and (ii) in the statement of the observation are satisfied.

Consider the function $f:\{\|\alpha\|: \alpha \in \mathcal{L}$ and $\forall \neg \alpha\} \rightarrow \mathcal{P}\left(\mathcal{M}_{\mathcal{L}}\right)$ defined by $f(\|\alpha\|)=f_{\mathbb{S}}(\alpha)$. Then it follows from Definition 2.2 that if $\alpha \in \mathcal{L}$ and $\forall \neg \alpha$ then $\emptyset \neq f(\|\alpha\|) \subseteq\|\alpha\|$.

Notice also that, it follows from the hypothesis that $B_{\mathbb{S}} \subseteq B$ and that (i) if $\alpha_{j} \in B_{\mathbb{S}}$ then $f\left(\left\|\neg \alpha_{k}\right\|\right)=f\left(\left\|\neg \alpha_{j}\right\|\right)$ or $f\left(\left\|\neg \alpha_{k}\right\|\right) \not \subset\left\|\neg \alpha_{j}\right\|$, for all $\alpha_{k} \in B_{\mathbb{S}}$; (ii) for all $\alpha_{l} \in B \cap \mathbf{K}$ there is some $\alpha_{m} \in B_{\mathbb{S}} \cap \mathbf{K}$ such that $f\left(\left\|\neg \alpha_{m}\right\|\right) \subseteq\left\|\neg \alpha_{l}\right\|$.

Hence, by Lemma A.3 we can conclude that $\left\{\mathcal{W} \in \mathbb{W}_{\mathbf{K} \perp B}: \mathcal{W} \subseteq\right.$ $\left.\bigcup_{\alpha_{i} \in B_{\mathbb{S}} \cap \mathbf{K}} f_{\mathbb{S}}\left(\neg \alpha_{i}\right)\right\} \neq \emptyset \quad$ and $\quad \bigcup\left\{\mathcal{W} \in \mathbb{W}_{\mathbf{K} \perp B}: \mathcal{W} \subseteq \bigcup_{\alpha_{i} \in B_{\mathbb{S}} \cap \mathbf{K}} f_{\mathbb{S}}\left(\neg \alpha_{i}\right)\right\}=$ $\bigcup_{\alpha_{i} \in B_{\mathbb{S}} \cap \mathbf{K}} f_{\mathbb{S}}\left(\neg \alpha_{i}\right)$.

Proofs

Proof of Theorem 3.4 Let $\div$ s be the operation defined by

$$
\mathbf{K} \div{ }_{\mathbb{S}} B=\left\{\begin{array}{ll}
T h\left(\|\mathbf{K}\| \cup\left(\bigcup_{\alpha_{i} \in B_{\mathbb{S}}} f_{\mathbb{S}}\left(\neg \alpha_{i}\right)\right)\right) & \text { if } B \cap C n(\emptyset)=\emptyset \\
\mathbf{K} & \text { if } B \cap C n(\emptyset) \neq \varnothing
\end{array},\right.
$$


where, for any finite set of sentences $B$ satisfying $B \cap \operatorname{Cn}(\emptyset)=\emptyset$, the set $B_{\mathbb{S}}$ is a subset of $B$ such that conditions (i)-(vi) in the statement of the theorem are satisfied. We must show that $\div_{\mathbb{S}}$ is a partial meet multiple contraction.

According to Observation 2.7, in order to prove that, it is enough to show that there is a propositional package selection function $h$ for $\|\mathbf{K}\|$ such that $\mathbf{K} \div{ }_{\mathbb{S}} B=T h\left(\|\mathbf{K}\| \cup\left(\bigcup h\left(\mathbb{W}_{\mathbf{K} \perp B}\right)\right)\right)$, for all sets $B$.

Let $h$ be such that $h\left(\mathbb{W}_{\mathbf{K} \perp B}\right)=\emptyset$ if $B \cap C n(\emptyset) \neq \emptyset$, and $h\left(\mathbb{W}_{\mathbf{K} \perp B}\right)=\{\mathcal{W} \in$ $\left.\mathbb{W}_{\mathbf{K} \perp B}: \mathcal{W} \subseteq \bigcup_{\alpha_{i} \in B_{\mathbb{S}} \cap \mathbf{K}} f_{\mathbb{S}}\left(\neg \alpha_{i}\right)\right\}$ if $B \cap C n(\emptyset)=\emptyset$.

We start by showing that $h$ is a function. Assume $B$ and $C$ are sets of sentences such that $\mathbb{W}_{\mathbf{K} \perp B}=\mathbb{W}_{\mathbf{K} \perp C}$. If $\mathbb{W}_{\mathbf{K} \perp B}=\mathbb{W}_{\mathbf{K} \perp C}=\emptyset$ then, according to Lemma A.1, $B \cap C n(\emptyset) \neq \emptyset$ and $C \cap C n(\emptyset) \neq \emptyset$ and, consequently, it follows from the definition of $h$ that $h\left(\mathbb{W}_{\mathbf{K} \perp B}\right)=h\left(\mathbb{W}_{\mathbf{K} \perp C}\right)=\emptyset$. Now we consider the case $\mathbb{W}_{\mathbf{K} \perp B}=\mathbb{W}_{\mathbf{K} \perp C} \neq \emptyset$. Again by Lemma A.1 we have that $B \cap C n(\emptyset)=C \cap$ $C n(\emptyset)=\emptyset$. Therefore, in order to demonstrate that $h\left(\mathbb{W}_{\mathbf{K} \perp B}\right)=h\left(\mathbb{W}_{\mathbf{K} \perp C}\right)$ we must show that $\left\{\mathcal{W} \in \mathbb{W}_{\mathbf{K} \perp B}: \mathcal{W} \subseteq \bigcup_{\alpha_{i} \in B_{\mathbb{S}} \cap \mathbf{K}} f_{\mathbb{S}}\left(\neg \alpha_{i}\right)\right\}=\left\{\mathcal{W} \in \mathbb{W}_{\mathbf{K} \perp C}: \mathcal{W} \subseteq\right.$ $\left.\bigcup_{\alpha_{i} \in C_{\mathbb{S}} \cap \mathbf{K}} f_{\mathbb{S}}\left(\neg \alpha_{i}\right)\right\}$. To do that we start by proving that $\bigcup_{\alpha_{i} \in B_{\mathbb{S}} \cap \mathbf{K}} f_{\mathbb{S}}\left(\neg \alpha_{i}\right)=$ $\bigcup_{\alpha_{i} \in C_{\mathbb{S}} \cap \mathbf{K}} f_{\mathbb{S}}\left(\neg \alpha_{i}\right)$. It follows from Lemma A.1 that $\mathbf{K} \perp B=\mathbf{K} \perp C$. Therefore, according to [9, Observation 1.39], it holds that every subset $X$ of $\mathbf{K}$ implies some element of $B$ if and only if $X$ implies some element of $C$.

Therefore, from (iii) we can conclude that $\bigcup_{\alpha_{i} \in\left(B_{N} \cap \mathbf{K}\right)_{\mathbb{S}}} f_{\mathbb{S}}\left(\neg \alpha_{i}\right)=$ $\bigcup_{\alpha_{i} \in\left(C_{N} \cap \mathbf{K}\right)_{\mathbb{S}}} f_{\mathbb{S}}\left(\neg \alpha_{i}\right)$. On the other hand it follows immediately from the definition of normalization of a set of sentences and from (v) that $\left(B_{N} \cap \mathbf{K}\right)_{\mathbb{S}}=\left((B \cap \mathbf{K})_{\mathbb{S}}\right)_{N}$ and $\left(C_{N} \cap \mathbf{K}\right)_{\mathbb{S}}=\left((C \cap \mathbf{K})_{\mathbb{S}}\right)_{N}$.

Hence, we obtain that $\bigcup_{\alpha_{i} \in\left((B \cap \mathbf{K})_{\mathbb{S})_{N}}\right.} f_{\mathbb{S}}\left(\neg \alpha_{i}\right)=\bigcup_{\alpha_{i} \in\left((C \cap \mathbf{K})_{\mathbb{S})_{N}}\right.} f_{\mathbb{S}}\left(\neg \alpha_{i}\right)$. So, (vi) gives us that $\bigcup_{\alpha_{i} \in(B \cap \mathbf{K})_{\mathbb{S}}} f_{\mathbb{S}}\left(\neg \alpha_{i}\right)=\bigcup_{\alpha_{i} \in(C \cap \mathbf{K})_{\mathbb{S}}} f_{\mathbb{S}}\left(\neg \alpha_{i}\right)$. Finally, we can conclude from (iv) that $\bigcup_{\alpha_{i} \in B_{\mathbb{S}} \cap \mathbf{K}} f_{\mathbb{S}}\left(\neg \alpha_{i}\right)=\bigcup_{\alpha_{i} \in C_{\mathbb{S}} \cap \mathbf{K}} f_{\mathbb{S}}\left(\neg \alpha_{i}\right)$, as required.

To show that $h$ is a propositional package selection function for $\|\mathbf{K}\|$ it only remains to prove that: (a) $h\left(\mathbb{W}_{\mathbf{K} \perp B}\right) \subseteq \mathbb{W}_{\mathbf{K} \perp B}$, and (b) if $\mathbb{W}_{\mathbf{K} \perp B} \neq \emptyset$ then $h\left(\mathbb{W}_{\mathbf{K} \perp B}\right) \neq \emptyset$.

(a) follows immediately from the definition of $h$. For (b), assume $\mathbb{W}_{\mathbf{K} \perp B} \neq \emptyset$. Then, by Lemma A.1, we have that $B \cap C n(\emptyset)=\emptyset$. So, according to the definition of $h, h\left(\mathbb{W}_{\mathbf{K} \perp B}\right)=\left\{\mathcal{W} \in \mathbb{W}_{\mathbf{K} \perp B}: \mathcal{W} \subseteq \bigcup_{\alpha_{i} \in B_{\mathbb{S}} \cap \mathbf{K}} f_{\mathbb{S}}\left(\neg \alpha_{i}\right)\right\}$ and (since $B_{\mathbb{S}} \subseteq B$ and conditions (i) and (ii) are satisfied) we can conclude from Lemma A.5 that $h\left(\mathbb{W}_{\mathbf{K} \perp B}\right) \neq \emptyset$.

Now it only remains to show that $\mathbf{K} \div{ }_{\mathbb{S}} B=T h\left(\|\mathbf{K}\| \cup\left(\bigcup h\left(\mathbb{W}_{\mathbf{K} \perp B}\right)\right)\right)$ for any set of sentences $B$.

Case $1, B \cap \operatorname{Cn}(\emptyset) \neq \emptyset$. Then, on the one hand, according to the definition of $\div_{\mathbb{S}}$, it holds that $\mathbf{K} \div{ }_{\mathbb{S}} B=\mathbf{K}$. And, on the other hand, it follows from the definition of $h$ that $T h\left(\|\mathbf{K}\| \cup\left(\bigcup h\left(\mathbb{W}_{\mathbf{K} \perp B}\right)\right)\right)=T h(\|\mathbf{K}\|)$. Hence, noticing that, according to [7, Property (1), p. 158], since we are assuming that $C n$ is compact, it holds that $T h(\|\mathbf{K}\|)=\mathbf{K}$, we can conclude that $\mathbf{K} \div{ }_{\mathbb{S}} B=$ $T h\left(\|\mathbf{K}\| \cup\left(\bigcup h\left(\mathbb{W}_{\mathbf{K} \perp B}\right)\right)\right)$, as required.

Case 2, $B \cap C n(\emptyset)=\emptyset$. Then $h\left(\mathbb{W}_{\mathbf{K} \perp B}\right)=\left\{\mathcal{W} \in \mathbb{W}_{\mathbf{K} \perp B}: \mathcal{W} \subseteq\right.$ $\left.\bigcup_{\alpha_{i} \in B_{\mathbb{S}} \cap \mathbf{K}} f_{\mathbb{S}}\left(\neg \alpha_{i}\right)\right\}$ and, according to the definition of $\div_{\mathbb{S}}$, we have that $\mathbf{K} \div \mathbb{S} B=T h\left(\|\mathbf{K}\| \cup\left(\bigcup_{\alpha_{i} \in B_{\mathbb{S}}} f_{\mathbb{S}}\left(\neg \alpha_{i}\right)\right)\right)$. Now, in order to show that the 
required identity holds we will prove that, indeed, the following stronger identity is satisfied: $\|\mathbf{K}\| \cup\left(\bigcup_{\alpha_{i} \in B_{\mathbb{S}}} f_{\mathbb{S}}\left(\neg \alpha_{i}\right)\right)=\|\mathbf{K}\| \cup\left(\bigcup h\left(\mathbb{W}_{\mathbf{K} \perp B}\right)\right)$.

First of all we must note that if $\alpha_{i} \notin \mathbf{K}$ then $f_{\mathbb{S}}\left(\neg \alpha_{i}\right) \subseteq\|\mathbf{K}\|$. Hence $\|\mathbf{K}\| \cup$ $\left(\bigcup_{\alpha_{i} \in B_{\mathbb{S}}} f_{\mathbb{S}}\left(\neg \alpha_{i}\right)\right)=\|\mathbf{K}\| \cup\left(\bigcup_{\alpha_{i} \in B_{\mathbb{S}} \cap \mathbf{K}} f_{\mathbb{S}}\left(\neg \alpha_{i}\right)\right)$. Finally, observing that it follows from Lemma A.5 that $\bigcup h\left(\mathbb{W}_{\mathbf{K} \perp B}\right)=\bigcup_{\alpha_{i} \in B_{\mathbb{S}} \cap \mathbf{K}} f_{\mathbb{S}}\left(\neg \alpha_{i}\right)$, we can conclude that $\|\mathbf{K}\| \cup\left(\bigcup_{\alpha_{i} \in B_{\mathbb{S}}} f_{\mathbb{S}}\left(\neg \alpha_{i}\right)\right)=\|\mathbf{K}\| \cup\left(\bigcup h\left(\mathbb{W}_{\mathbf{K} \perp B}\right)\right)$ as we wished to prove.

Proof of Observation 3.6 (a) follows immediately from the fact that, for all $l \in$ $\{1, \ldots, m\}$, given $\alpha_{i}, \alpha_{j} \in C_{l}^{\prime}$, it holds that if $f_{\mathbb{S}}\left(\neg \alpha_{i}\right) \nsubseteq f_{\mathbb{S}}\left(\neg \alpha_{j}\right)$ then $f_{\mathbb{S}}\left(\neg \alpha_{i}\right) \not \subset$ $\left\|\neg \alpha_{j}\right\|$ (because $\mathbb{S}_{\neg \alpha_{i}}=\mathbb{S}_{\neg \alpha_{j}}$ ).

On the other hand, (b) is an immediate consequence of the fact that, if $m>1$ and $l \in\{2, \ldots, m\}$ then from $\alpha_{i} \in C_{l}$ and $\alpha_{j} \in B_{l-1}$ it follows that $\mathbb{S}_{\neg \alpha_{i}} \subset \mathbb{S}_{\neg \alpha_{j}}$ and, consequently, $f_{\mathbb{S}}\left(\neg \alpha_{j}\right) \neq f_{\mathbb{S}}\left(\neg \alpha_{i}\right)$.

\section{Proof of Observation 3.7}

(i) If $B \subseteq \operatorname{Cn}(\emptyset)$ it follows trivially, since by Definition 3.5, $B_{\mathbb{S}}=$ $\emptyset$. Let $B \backslash C n(\emptyset) \neq \emptyset$ and $C_{1}, \ldots, C_{m}, C_{1}^{\prime}, \ldots, C_{m}^{\prime}, C_{1}^{\prime \prime}, \ldots, C_{m}^{\prime \prime}$, $B_{1}, \ldots, B_{m}$ be the subsets of $B$ described in Definition 3.5. Let $\alpha_{j} \in B_{\mathbb{S}}$ and assume by reductio that there is some $\alpha_{i} \in B_{\mathbb{S}}$ such that $f_{\mathbb{S}}\left(\neg \alpha_{i}\right) \neq f_{\mathbb{S}}\left(\neg \alpha_{j}\right)$ and $f_{\mathbb{S}}\left(\neg \alpha_{i}\right) \subset\left\|\neg \alpha_{j}\right\|$. From the latter condition and Definition 2.2 it follows that $\mathbb{S}_{\neg \alpha_{j}} \subseteq \mathbb{S}_{\neg \alpha_{i}}$.

Case $1, \mathbb{S}_{\neg \alpha_{j}}=\mathbb{S}_{\neg \alpha_{i}}$. Then, there is some class $C_{l}$ such that $\alpha_{j}, \alpha_{i} \in$ $C_{l}$. Since $\alpha_{j}, \alpha_{i} \in B_{\mathbb{S}}$ we must have $\alpha_{j}, \alpha_{i} \in C_{l}^{\prime}$. But, in that case, from $f_{\mathbb{S}}\left(\neg \alpha_{i}\right) \neq f_{\mathbb{S}}\left(\neg \alpha_{j}\right)$ and $f_{\mathbb{S}}\left(\neg \alpha_{i}\right) \subset\left\|\neg \alpha_{j}\right\|$ we conclude that $\alpha_{j} \notin C_{l}^{\prime \prime}$ (cf. Observation 3.6-(a)), which contradicts $\alpha_{j} \in B_{\mathbb{S}}$.

Case 2.2, $\mathbb{S}_{\neg \alpha_{j}} \subset \mathbb{S}_{\neg_{\alpha_{i}}}$. Then, there are two classes $C_{l}$ and $C_{m}$, with $l<m$ such that $\alpha_{i} \in C_{l}$ and $\alpha_{j} \in C_{m}$. Since $\alpha_{i} \in B_{\mathbb{S}}$ we have that $\alpha_{i} \in C_{l}^{\prime \prime}$. Hence, from $C_{l}^{\prime \prime} \subseteq B_{l} \subseteq B_{m-1}$ we can conclude that $\alpha_{i} \in$ $B_{m-1}$. But then, from $f_{\mathbb{S}}\left(\neg \alpha_{i}\right) \neq f_{\mathbb{S}}\left(\neg \alpha_{j}\right)$ and $f_{\mathbb{S}}\left(\neg \alpha_{i}\right) \subset\left\|\neg \alpha_{j}\right\|$ it follows that $\alpha_{j} \notin C_{m}^{\prime}$ (cf. Observation 3.6-(b)), which contradicts $\alpha_{j} \in B_{\mathbb{S}}$.

(ii) We will prove that the following condition (stronger) than (ii) holds: If $\alpha_{j} \in B \backslash B_{\mathbb{S}}$, then there is some $\alpha_{l} \in B_{\mathbb{S}}$ such that $f_{\mathbb{S}}\left(\neg \alpha_{l}\right) \subset\left\|\neg \alpha_{j}\right\|$ and $f_{\mathbb{S}}\left(\neg \alpha_{l}\right) \neq f_{\mathbb{S}}\left(\neg \alpha_{j}\right)$.

Let $\alpha_{j} \in B \backslash B_{\mathbb{S}}$ and let $C_{1}, \ldots, C_{m}, C_{1}^{\prime}, \ldots, C_{m}^{\prime}, C_{1}^{\prime \prime}, \ldots, C_{m}^{\prime \prime}$, $B_{1}, \ldots, B_{m}$ be the subsets of $B$ considered in the process of construction of the set $B_{\mathbb{S}}$ described in Definition 3.5.

We will consider separately the two possibilities $\alpha_{j} \in C_{1}$ or $\alpha_{j} \in$ $C_{n}$, with $n>1$.

Case $1, \alpha_{j} \in C_{1}$. Then, since by construction $C_{1}=C_{1}^{\prime}, \alpha_{j} \in C_{1}^{\prime}$. It follows from $\alpha_{j} \notin B_{\mathbb{S}}$ that $\alpha_{j} \notin C_{1}^{\prime \prime}$. Hence, there is some $\alpha_{k} \in C_{1}^{\prime}$ such that $f_{\mathbb{S}}\left(\neg \alpha_{k}\right) \subset f_{\mathbb{S}}\left(\neg \alpha_{i}\right)$. We have that for any $\alpha_{r}, \alpha_{s} \in C_{1}^{\prime}$ it holds that $\mathbb{S}_{\neg \alpha_{r}}=\mathbb{S}_{\neg \alpha_{s}}$. Then, according to Lemma A.4, there is some $\alpha_{l} \in C_{1}^{\prime}$ such that $f_{\mathbb{S}}\left(\neg \alpha_{l}\right) \subset f_{\mathbb{S}}\left(\neg \alpha_{j}\right)$ and for all $\alpha_{m} \in C_{1}^{\prime}$ it holds that $f_{\mathbb{S}}\left(\neg \alpha_{m}\right) \not \subset f_{\mathbb{S}}\left(\neg \alpha_{l}\right)$. Hence $\alpha_{l} \in C_{1}^{\prime \prime}$ and, consequently, 
$\alpha_{l} \in B_{\mathbb{S}}$. Finally we note that from $f_{\mathbb{S}}\left(\neg \alpha_{l}\right) \subset f_{\mathbb{S}}\left(\neg \alpha_{j}\right)$ it follows immediately that $f_{\mathbb{S}}\left(\neg \alpha_{l}\right) \subset\left\|\neg \alpha_{j}\right\|$ and $f_{\mathbb{S}}\left(\neg \alpha_{l}\right) \neq f_{\mathbb{S}}\left(\neg \alpha_{j}\right)$ as it was required.

Case $2, \alpha_{j} \in C_{n}$, with $1<n \leq m$. From $\alpha_{j} \notin B_{\mathbb{S}}$ it follows that $\alpha_{j} \notin C_{n}^{\prime \prime}$, and we have to consider the two possibilities $\alpha_{j} \notin C_{n}^{\prime}$ or $\alpha_{j} \in C_{n}^{\prime}$.

Case 2.1, $\alpha_{j} \notin C_{n}^{\prime}$. Then there is some $\alpha_{l} \in B_{n-1}$ such that $f_{\mathbb{S}}\left(\neg \alpha_{l}\right) \subset\left\|\neg \alpha_{j}\right\|$. Since $B_{n-1} \subseteq B_{\mathbb{S}}$ we have that $\alpha_{l} \in B_{\mathbb{S}}$.

On the other hand, it follows from the construction of $B_{n-1}$ and $C_{n}$ that $\mathbb{S}_{\neg \alpha_{j}} \subset \mathbb{S}_{\neg \alpha_{l}}$ and, therefore, according to Definition 2.2 it holds that $f_{\mathbb{S}}\left(\neg \alpha_{l}\right) \cap \mathbb{S}_{\neg \alpha_{j}}=\emptyset$. Hence, since $\emptyset \neq f_{\mathbb{S}}\left(\neg \alpha_{j}\right) \subseteq \mathbb{S}_{\neg \alpha_{j}}$, we can conclude that $f_{\mathbb{S}}\left(\neg \alpha_{l}\right) \neq f_{\mathbb{S}}\left(\neg \alpha_{j}\right)$, and this finishes the proof.

Case 2.2, $\alpha_{j} \in C_{n}^{\prime}$. Then, since $\alpha_{j} \notin C_{n}^{\prime \prime}$, there is some $\alpha_{k} \in C_{n}^{\prime}$ such that $f_{\mathbb{S}}\left(\neg \alpha_{k}\right) \subset f_{\mathbb{S}}\left(\neg \alpha_{j}\right)$. Reasoning as we did in Case 1 above we can conclude that there is some $\alpha_{l} \in B_{\mathbb{S}}$ such that $f_{\mathbb{S}}\left(\neg \alpha_{l}\right) \subset$ $\left\|\neg \alpha_{j}\right\|$ and $f_{\mathbb{S}}\left(\neg \alpha_{l}\right) \neq f_{\mathbb{S}}\left(\neg \alpha_{j}\right)$ as we wished to prove.

(iii) Assume every subset $X$ of $\mathbf{K}$ implies some element of $B$ if and only if $X$ implies some element of $C$. According to Lemma A.2-(ii) it holds that for all $\beta_{i} \in B_{N} \cap \mathbf{K}$ there is some $\varsigma_{i} \in$ $C_{N} \cap \mathbf{K}$ such that $\left\|\beta_{i}\right\|=\left\|\varsigma_{i}\right\|$. Hence it follows trivially from the definition of $\mathbb{S}$-based filtration of a set of sentences that for all $\beta_{i} \in\left(B_{N} \cap \mathbf{K}\right)_{\mathbb{S}}$ there is some $\varsigma_{i} \in\left(C_{N} \cap \mathbf{K}\right)_{\mathbb{S}}$ such that $\left\|\beta_{i}\right\|=\left\|\varsigma_{i}\right\|$, and we are done.

(iv) and (v) Follow immediately from the definitions of normalization and of S-based filtration of a set of sentences.

(vi) That $\bigcup_{\alpha_{i} \in\left(B_{\mathbb{S}}\right)_{N}} f_{\mathbb{S}}\left(\neg \alpha_{i}\right) \subseteq \bigcup_{\alpha_{i} \in B_{\mathbb{S}}} f_{\mathbb{S}}\left(\neg \alpha_{i}\right)$ follows immediately from $\left(B_{\mathbb{S}}\right)_{N} \subseteq B_{\mathbb{S}}$. For the converse inclusion, assume $M \in$ $\bigcup_{\alpha_{i} \in B_{\mathbb{S}}} f_{\mathbb{S}}\left(\neg \alpha_{i}\right)$ then there is some $\alpha_{j} \in B_{\mathbb{S}}$ such that $M \in f_{\mathbb{S}}\left(\neg \alpha_{j}\right)$. If $\alpha_{j} \in\left(B_{\mathbb{S}}\right)_{N}$ it follows immediately that $M \in \bigcup_{\alpha_{i} \in\left(B_{\mathbb{S}}\right)_{N}} f_{\mathbb{S}}\left(\neg \alpha_{i}\right)$ and we are done. Now assume $\alpha_{j} \notin\left(B_{\mathbb{S}}\right)_{N}$. It follows from Lemma A.2-(i) that there is some $\alpha_{k} \in\left(B_{\mathbb{S}}\right)_{N}$ such that $\left\|\neg \alpha_{k}\right\| \subset$ $\left\|\neg \alpha_{j}\right\|$. Hence we have that $\alpha_{j}, \alpha_{k} \in B_{\mathbb{S}}$ and $f_{\mathbb{S}}\left(\neg \alpha_{k}\right) \subset\left\|\neg \alpha_{j}\right\|$. So we obtain from (i) (already proven above) that $f_{\mathbb{S}}\left(\neg \alpha_{k}\right)=$ $f_{\mathbb{S}}\left(\neg \alpha_{j}\right)$. Therefore $M \in f_{\mathbb{S}}\left(\neg \alpha_{k}\right)$ and we can conclude that $M \in$ $\bigcup_{\alpha_{i} \in\left(B_{\mathbb{S}}\right)_{N}} f_{\mathbb{S}}\left(\neg \alpha_{i}\right)$.

Proof of Theorem 3.9 Follows immediately from Theorem 3.4 and Observation 3.7.

\section{References}

1. Alchourrón, C., \& Makinson, D. (1981). Hierarchies of regulations and their logic. In R. Hilpinen (Ed.), New studies in Deontic logic: Norms, actions, and the foundations of ethics (pp. 125-148). Amsterdam: Reidel. 
2. Alchourrón, C., Gärdenfors, P., \& Makinson, D. (1985). On the logic of theory change: Partial meet contraction and revision functions. Journal of Symbolic Logic, 50, 510-530.

3. Fermé, E., Saez, K., Sanz, P. (2003). Multiple kernel contraction. Studia Logica, 73, 183-195.

4. Fuhrmann, A. (1988). Relevant logics, modal logics and theory change. $\mathrm{PhD}$ thesis, Australian National University, Camberra.

5. Fuhrmann, A. (1991). Theory contraction through base contraction. Journal of Philosophical Logic, 20, 175-203.

6. Fuhrmann, A., \& Hansson, S. O. (1994). A survey of multiple contraction. Journal of Logic, Language and Information, 3, 39-74.

7. Grove, A. (1988). Two modellings for theory change. Journal of Philosophical Logic, 17, 157170.

8. Hansson, S. O. (1989). New operators for theory change. Theoria, 55, 114-132.

9. Hansson, S. O. (1999). A textbook of belief dynamics. Theory change and database updating. Applied logic series (Vol. 11). Dordrecht: Kluwer.

10. Reis, M. D. L., \& Fermé, E. (2011). Possible worlds semantics for partial meet multiple contraction. Journal of Philosophical Logic. doi:10.1007/s10992-011-9198-y.

11. Spohn, W. (2010). Multiple contraction revisited. In M. Suárez, M. Dorato, \& M. Rédei (Eds.), EPSA epistemology and methodology of science (pp. 279-288). Netherlands: Springer.

12. Zhang, D. (1996). Belief revision by sets of sentences. Journal of Computer Science and Technology, 11(2), 108-125.

13. Zhang, D., \& Foo, N. (2001). Infinitary belief revision. Journal of Philosophical Logic, 30(6), $525-570$. 